\title{
Histopathological Heterogeneity of Neuropathy in Insulin-dependent and Non-insulin-dependent Diabetes, and Demonstration of Axo-glial Dysjunction in Human Diabetic Neuropathy
}

\author{
Anders A. F. Sima, Virgil Nathaniel, Vera Bril,* Thomas A. J. McEwen, and Douglas A. Greene \\ Neuropathology Research Laboratory, Department of Pathology, University of Manitoba, Winnipeg, Manitoba, R3E 0W3 Canada; \\ *Department of Medicine, University of Toronto, Toronto, Ontario, M5G 1 L7 Canada; and ${ }^{\ddagger}$ Diabetes Research and Training Center \\ and Department of Internal Medicine, University of Michigan Medical Center, Ann Arbor, Michigan 48109
}

\begin{abstract}
Altered sorbitol and myo-inositol metabolism, (Na,K)-ATPase function, electrochemical sodium gradients, axonal swelling, and distortion and disruption of the node of Ranvier ("axo-glial dysjunction") directly implicate hyperglycemia in the pathogenesis of neuropathy in diabetic rats, but the relevance of this sequence to clinical neuropathy in heterogeneous groups of diabetic patients remains to be established. Fascicular sural nerve morphometry in 11 patients with neuropathy complicating insulin-dependent diabetes revealed a pattern of interrelated structural changes strikingly similar to that of the diabetic rat when compared to age-matched controls. 17 older non-insulin-dependent diabetic patients with comparable duration and severity of hyperglycemia and severity of neuropathy, displayed similar nerve fiber loss, paranodal demyelination, paranodal remyelination and segmental demyelination compared to age-matched controls, but axo-glial dysjunction was replaced by Wallerian degeneration as the primary manifestation of fiber damage, and fiber loss occurred in a spatial pattern consistent with an ischemic component. The mechanistic model developed from the diabetic rat does indeed appear to apply to human diabetic neuropathy, but superimposed hormonal, metabolic, vascular, and/or age-related effects alter the morphologic expression of the neuropathy in non-insulin dependent diabetes.
\end{abstract}

\section{Introduction}

Insulin-dependent (IDDM) ${ }^{1}$ and non-insulin-dependent (NIDDM) diabetes mellitus is the most common cause of neuropathy in the Western world $(1,2)$. The distal symmetric polyneuropathy associated with diabetes is presumed to result

This work was presented in part at the American Society for Clinical Investigation, Washington, DC in May 1986 and at the annual meeting of the American Neuropathology Association, Minneapolis, $\mathrm{MN}$ in June 1986.

Address reprint requests to Dr. Greene.

Received for publication 18 February 1987 and in revised form 20 July 1987.

1. Abbreviations used in this paper: BB, Bio-breeding (rat); CV, coefficient of variation of myelinated fiber density; IDDM, insulin-dependent diabetes mellitus; NIDDM, non-insulin-dependent diabetes mellitus.

J. Clin. Invest.

(c) The American Society for Clinical Investigation, Inc.

0021-9738/88/02/0349/16 \$2.00

Volume 81, February 1988, 349-364 from a complex interplay between metabolic factors related to hyperglycemia and unidentified independent genetic and environmental variables (1, 3-6). The conversion of excess glucose to sorbitol by the enzyme aldose reductase and the resulting depletion of myo-inositol and its phosphoinositide metabolites, which in selected cells leads to inactivation of the $(\mathrm{Na}, \mathrm{K})-\mathrm{ATPase}$, is postulated to play an important pathogenetic role $(3,6)$.

The spatial distribution of these linked metabolic defects does not convincingly localize the primary pathogenetic processes of this disorder to any of the multiple cell types in peripheral nerve. Aldose reductase is primarily confined to paranodal Schwann cell cytoplasm and vascular endothelial cells in nerve (7), whereas elevated ambient glucose levels appear to depress myo-inositol and $(\mathrm{Na}, \mathrm{K})$-ATPase in peripheral neurons $(6,8-12)$ as well as vascular smooth muscle and perhaps endothelial cells $(13,14)$.

Similarly, numerous morphological and electrophysiological analyses of human diabetic neuropathy have emphasized entirely different lesions involving peripheral nerve axons, Schwann cells, perineurial cells, or endoneurial vascular elements in the pathogenesis of diabetic neuropathy $(1,15-36)$. The proximal-to-distal increase in morphometric abnormalities $(1,20)$ and the topographic and temporal distribution of neurological signs and symptoms in diabetic distal symmetric polyneuropathy suggest a primary axonopathy preferentially involving longer myelinated axons (31-33). Nerve biopsies from young diabetic patients characteristically exhibit ultrastructural lesions most consistent with an early primary distal axonal atrophy and degeneration $(28,29,35,37)$. Yet studies of sural nerve biopsies by Thomas and Lascelles $(24,25)$ and others $(27,30)$, and autopsy studies (26) have also emphasized segmental demyelination and remyelination in diabetic distal symmetric polyneuropathy, postulating a primary abnormality of Schwann cells. Endoneurial vascular abnormalities such as basement membrane thickening and reduplication, endothelial cell swelling and proliferation, and platelet aggregation resulting in vessel occlusion have been noted in sural nerve biopsies $(21,23)$ and at autopsy $(1,17,20,22,36)$ of diabetic patients. A quantitative increase in these vascular abnormalities in association with focal loss of myelinated fibers in older diabetic subjects has been interpreted to suggest hypoxic or ischemic damage to nerve fibers in diabetic distal symmetric polyneuropathy $(1,21,23)$. Thus, while demonstrating that most tissue elements of peripheral nerve are involved in the disease process at some point, existing studies of human diabetic distal symmetric polyneuropathy provide no consistent evidence as to the location of the initial inciting events.

In contrast, recent studies of diabetic animal models localize prominent early structural and functional abnormalities to 
the specialized interface between axons and Schwann cells at the nodes of Ranvier of myelinated nerve fibers. Soon after the onset of acute spontaneous diabetes in the Bio-breeding (BB) rat, nerve conduction is reversibly slowed by a diminished nodal equilibrium potential owing to intraaxonal sodium accumulation attributable to impaired (Na,K)-ATPase activity $(8,9,38,39)$. This defect in $(\mathrm{Na}, \mathrm{K})$-ATPase in turn results from the myo-inositol depletion that accompanies the accumulation of sorbitol (40). Intraaxonal sodium accumulation is associated with nodal and paranodal swelling, and spatial deformation of the paranodal apparatus $(39,40)$. Persistent nodal deformation has been speculated to contribute to a poorly reversible disruption and loss of strategic junctional complexes between terminal loops of myelin and the paranodal axolemma (41). This "axo-glial dysjunction" correlates with the more chronic and poorly reversible slowing of nerve conduction $(9,41,42)$ that is attributable to a marked decrease in nodal sodium permeability $(9,39)$, which in turn probably reflects the escape of nodal sodium channels by lateral migration into the internode through the now-damaged paranodal junctional barrier $(39,41,43)$. Axo-glial dysjunction also most likely constitutes the initial stage in the development of paranodal demyelination (39) and is followed by a progressive but subtle diminution of axon cylinder size or "axonal atrophy" (44). This complex set of interrelated metabolic, biophysical, and ultrastructural processes first expressed at the highly specialized interface between the axon and its associated Schwann cells at the node of Ranvier may constitute a primary pathogenetic element in the neuropathy of this animal model for IDDM.

In order to assess the applicability of this model to the pathogenesis of the neuropathy complicating IDDM or NIDDM in human subjects, and thereby gain further insight into the pathogenesis of human diabetic neuropathy, sural nerve biopsies obtained from diabetic patients with distal symmetric polyneuropathy entering an aldose reductase inhibitor trial were examined for characteristic structural alterations at the node of Ranvier. Because elements of the metabolic sequence involving sorbitol, myo-inositol, and the (Na,K)ATPase probably exist in vascular as well as neural components of peripheral nerve, focal loss of nerve fibers was assessed as a possible expression of ischemic nerve damage $(1,20,21)$ in these nerve biopsies. Finally, because some forms of vascular disease may be primarily age-related in diabetic subjects, and may differ between patients with IDDM and NIDDM (45), biopsies obtained from patients with IDDM and NIDDM were analyzed separately and compared to sural nerve biopsies from carefully age-matched controls.

These studies confirm, for the first time, lesions at the node of Ranvier and subtle axonal atrophy in human patients with peripheral neuropathy complicating IDDM that are characteristic of the neuropathy in the BB rat model. In patients with NIDDM, despite similar degrees of nerve fiber loss, paranodal swelling, and paranodal demyelination compared to their agematched controls, axo-glial dysjunction was not increased, whereas Wallerian degeneration was significantly more prevalent. Focal loss of nerve fibers was related to age in both control subjects and IDDM patients but was statistically significantly increased only in nerve biopsies from patients with NIDDM compared with age-matched controls as a function of duration of diabetes. These findings are interpreted to support the applicability of the BB rat model to the pathogenesis of human diabetic neuropathy, especially in IDDM where paranodal swelling, axo-glial dysjunction, paranodal demyelination, and paranodal remyelination correlated closely, but suggest that the expression of metabolic damage may be more complex in NIDDM presumably due to effects of age or associated vascular disease.

\section{Methods}

Patient selection criteria. All patients enrolled at the Toronto clinical center in a 12-mo prospective, randomized, double-blind, multicenter clinical trial of the aldose reductase inhibitor sorbinil in the treatment of symptomatic diabetic polyneuropathy were requested to undergo a diagnostic fascicular sural nerve biopsy as part of the baseline screening process. Patients were also informed that they might be requested to undergo a second fascicular biopsy at the end of the trial to evaluate possible effects of treatment. Entrance requirements were age 18-65 $\mathrm{yr}$, neuropathy of 6 mo or more duration, and IDDM or NIDDM as defined by the National Diabetes Data Group criteria (46) of 1-20 yr in duration with a current hemoglobin $A_{1} C$ greater than the 95th percentile of the nondiabetic adult population. Neuropathy was defined by symptoms or objective physical findings consistent with a distal symmetric polyneuropathy, confirmed by the presence of either elevated tactile (47) or thermal (48) perception thresholds, or slowed motor nerve conduction velocity (peroneal $<40 \mathrm{M} \cdot \mathrm{s}^{-1}$ if age $<40 \mathrm{yr}$ or $<37$ $\mathrm{M} \cdot \mathrm{s}^{-1}$ if age $\geq 40 \mathrm{yr}$ ). Women with childbearing potential or patients with systemic conditions other than diabetes associated with peripheral neuropathy were excluded, as were patients with symptomatic peripheral vascular disease or renal or hepatic disease. Patients were not enrolled in the trial if their diabetic treatment regimen or degree of metabolic control was substantially altered within the preceding 3 mo.

Patient populations. 28 of 31 patients with diabetic neuropathy enrolled at the Toronto clinical center consented to and successfully underwent baseline fascicular sural nerve biopsies without complication. Patients were classified as having IDDM or NIDDM according to the National Diabetes Data Group clinical guidelines (46) by one of the investigators (Dr. Bril) unaware of biopsy results. 11 patients, seven men and four women, with a mean age of $38.5 \pm 4.4$ (SEM) yr (range 22-61 yr) and a mean duration of diabetes of $11 \pm 1.6 \mathrm{yr}$ (range 1-19 yr) were classified as having IDDM. 17 patients, 16 men and one woman, with a mean age of $56.1 \pm 2.0 \mathrm{yr}$ (range $44-65 \mathrm{yr}, P<0.005 \mathrm{vs.} \mathrm{IDDM}$ patients) and a mean duration of $10.4 \pm 1.1$ yr (range 2-18 yr) were classified as having NIDDM. All IDDM and 13 NIDDM patients were treated with insulin, and four NIDDM patients were treated with sulfonylureas. Mean hemoglobin $A_{1} C$ was $8.6 \pm 0.7 \%$ (range $6.1-12.8 \%$ ) in patients with IDDM, and $8.0 \pm 0.3 \%$ (range $6.3-10.5 \%$ ) in patients with NIDDM. Mean duration of symptomatic neuropathy was $3.04 \pm 1.02$ yr (range $0.75-10.00 \mathrm{yr}$ ) in patients with IDDM, and $2.72 \pm 0.70$ years (range 0.42-9.00 yr) in patients with NIDDM. Sural nerve specimens from 19 patients with no history of diabetes or neuropathy were obtained either in conjunction with cadaveric organ donation, or at autopsy within $6 \mathrm{~h}$ of death, and used as control material. Preliminary experiments, in which paired fascicular biopsies were obtained from the same donors during organ donation and at subsequent autopsy revealed that neither myelinated fiber morphometry nor teased fiber pattern were changed during the intervening postmortem period.

Electrophysiological studies. Nerve conduction studies were repeated four times during an 8-wk baseline and placebo "run-in" period at the beginning of the trial. These included measurements of antidromic median and sural sensory conduction velocity and compound action potential amplitude, and orthodromic median and peroneal motor conduction velocity and evoked muscle potential amplitude, using surface electrodes placed on the patients' dominant side, and performed in a temperature-controlled room at $34^{\circ} \mathrm{C}$. For sural nerve conduction measurements, the active recording electrode was placed over the sural nerve at the level of the lower tip of the lateral malleolus, and the reference electrode placed $3 \mathrm{~cm}$ distal to the active electrode, 
below and lateral to the malleolus. The stimulating cathode was placed $14 \mathrm{~cm}$ proximal to the active recording electrode slightly lateral to the midline in the lower third of the leg, with the anode proximal to the cathode. The ground electrode was placed between the cathode and the active recording electrode. A stimulus of 0.2 -ms duration with an intensity equal to $120 \%$ of that yielding a maximal response was applied via the stimulating electrode, and the resulting compound action potential was recorded using averaging techniques. Median and peroneal conduction studies were also performed in a standardized manner per the experimental protocol.

Surgical procedure and specimen preparation. A single fascicular biopsy 5-6 cm in length was obtained surgically under local anesthesia from the sural nerve just posterior to the lateral malleolus. The specimens were divided transversely into four equally sized portions, the two most proximal of which were immediately fixed in cacodylatebuffered $2.5 \%$ glutaraldehyde for $24 \mathrm{~h}$, postfixed for $2 \mathrm{~h}$ at $4^{\circ} \mathrm{C}$ in $1 \%$ cacodylate-buffered osmium tetroxide (pH 7.4), and were employed for morphometric studies (the two distal segments were immediately snap-frozen in liquid nitrogen for biochemical studies to be reported separately) (49). The two proximal segments were both dehydrated in graded concentrations of alcohol; one was embedded in Epon, and cross and longitudinal sections were used for morphometric analysis, and the other was used for teased fiber preparations in Epon. Thin sections for electron-microscopic examination were stained with aqueous uranyl acetate and lead citrate. All morphometric analyses were performed by one author (Dr. Nathaniel) and all teased fiber analyses by another (Dr. Sima), each of whom were unaware of the source of the biopsy.

Morphometric techniques. The myelinated fiber number, size, and spatial distribution were characterized on semithin $(0.5 \mu \mathrm{M})$ toluidine blue-stained cross sections of sural nerve fascicles photographed at a total magnification of 1,600 . The area of each myelinated fiber of the entire fascicle was measured from approximately 20 photographs per biopsy with the aid of a 9872A HP digitizer interfaced with a 9825A desk computer and plotter (Hewlett-Packard Co., Cupertino, CA) for the construction of histograms of myelinated fiber size, and for the calculation of mean fiber size for each individual biopsy. Endoneurial area was digitized from prints with a total magnification of 250 , and myelinated fiber density (number of fibers per unit area of endoneurial space) and occupancy (percent endoneurial area occupied by myelinated fibers) were calculated as previously described in detail (44). The intrafascicular coefficient of variation of myelinated fiber density (CV), a measure of the nonuniformity of the spatial distribution of myelinated nerve fibers over the cross-sectional area of each fascicle, has been interpreted to reflect hypoxic or ischemic nerve damage (50). Each fascicle was divided into five $72^{\circ}$ radial sectors, and the mean density of myelinated fibers in each sector was determined. CV was defined in this study as the quotient of the standard deviation of sectorial fiber densities and the mean sectorial fiber density of each fascicle.

The axon-myelin ratio, defined as the ratio of the natural logarithm of the axonal cross-sectional area to the number of surrounding myelin lamellae as recommended by O'Neill and Gilliatt (51), was determined for a mean of $43.7 \pm 1.4$ randomly chosen fibers for each fascicle on electronmicroscopic prints at a magnification of 27,420 (44). This normally linear relationship, as defined by linear regression analysis, decreases under circumstances of axonal atrophy and increases when demyelinated or regenerating fibers are in the process of remyelination.

The frequency of axo-glial dysjunction (41), defined as the percentage of paranodal terminal loops of myelin not exhibiting the characteristic junctional complexes at their abutment with the axolemma (43), was determined on serial longitudinal electromicroscopic sections at a magnification of 39,170 . In order to avoid repeated analyses of the same node in serial sections, each examined node was photographed and coded for identification. In each fascicle, $391 \pm 32$ terminal loops in $12 \pm 0.2$ nodes of Ranvier (minimum of 10 ) with a clearly discernible trilamellar structure to both the axolemmal and myelin membranes were examined for the presence of discrete junctional complexes as previously described in detail (41).

Internodal length-diameter ratio was measured from single teased fibers using an eyepiece caliper. The internodal diameter was measured at five equidistant points along the internode, with the mean diameter used as a measure of overall fiber diameter. The internodal length-diameter ratio was calculated by linear regression analysis.

Teased fiber analysis. The spectrum and pattern of abnormal myelinated nerve fibers was assessed by analysis of teased fibers. A mean of $67 \pm 7$ randomly teased single fibers from each biopsy were examined by phase-contrast light microscopy at a magnification of 480 . Each fiber was qualitatively assigned to one of eight categories based on the presence or absence of characteristic structural features according to a modification of the techniqe described by Dyck et al. (52): A, normal fibers; $B$, paranodal swelling defined as a paranodal diameter $>150 \%$ of the internodal diameter; $C$, paranodal demyelination; $D$, excessive myelin wrinkling; $\mathrm{E}$, intercalated (remyelinated) nodes; $\mathrm{F}$, Wallerian degeneration; $\mathbf{G}$, segmental demyelination; $H$, regenerated and remyelinated fibers. The frequencies of abnormalities were expressed as a percentage of fibers examined.

Statistical analysis. Results were generally expressed as a mean \pm standard error of the mean (SEM) with the significance of differences between groups calculated by the $t$ test, except that morphometric parameters were expressed as estimated means and observed ranges with significance of differences between groups calculated by analysis of variation (ANOVA). Morphometric data were also corrected for age and assessed for significance of differences between groups on the basis of analysis of covariance. Multiple linear regression analysis was performed by the method of least squares using age, duration of diabetes, and hemoglobin $\mathrm{A}_{1} \mathrm{C}$ as independent variables. Comparisons of distributions of myelinated fiber size were performed using chi-square distribution.

\section{Results}

Neurological function. As required by the entrance criteria, all patients exhibited clinical signs and/or symptoms of peripheral neuropathy. Sural nerve sensory conduction velocity and amplitude of the evoked compound action potential were markedly depressed compared with the age-corrected values for a nondiabetic reference population (53): $37.8 \pm 0.6 \mathrm{M} \cdot \mathrm{s}^{-1} \mathrm{com}$ pared with $53.7 \pm 0.4 \mathrm{M} \cdot \mathrm{s}^{-1}, P<0.001$; and $3.0 \pm 0.4 \mu \mathrm{V}$ compared with $10.0 \pm 0.1 \mu \mathrm{V}, P<0.001$. Similar abnormalities were noted in the other electrophysiological parameters and in tactile and thermal thresholds, which will be reported elsewhere (54).

There were no differences in these parameters between subjects with IDDM and NIDDM, with the exception that two IDDM patients but no NIDDM patients had unmeasurable peroneal motor conduction. Sural nerve conduction velocity and compound action potential amplitudes were, respectively, $37.4 \pm 1.0 \mathrm{M} \cdot \mathrm{s}^{-1}$ and $2.9 \pm 1.0 \mu \mathrm{V}$ in patients with IDDM, and $38.1 \pm 1.0 \mathrm{M} \cdot \mathrm{s}^{-1}$ and $3.0 \pm 0.8 \mu \mathrm{V}$ in patients with IDDM. Thus, IDDM and NIDDM patients, though differing in age, were similar in duration and severity of hyperglycemia and neuropathy as measured by objective parameters in the context of this clinical trial.

Myelinated fiber number. Myelinated fiber density and occupancy in fascicular biopsies of sural nerve reflect the total number of sural nerve myelinated fibers to the extent that a representative fascicle is biopsied. Total fascicular area does not change as a result of diabetes (20) and mean total endoneurial area of the biopsied fascicles were similar in the two diabetic groups and their respective age-matched controls 
(data not shown). Fiber density ( $n$ fibers $\cdot \mathrm{mm}^{-2}$ ) and occupancy (percent total fascicular endoneurial cross-sectional area occupied by myelinated fibers) were reduced by $\sim 50 \%$ in IDDM and NIDDM patients compared with their respective age-matched controls (Table I $A$, columns 1 and 2). Although fiber density and occupancy were numerically smaller in the older controls for the NIDDM patients than in the younger IDDM controls, this difference achieved statistical significance for fiber occupancy alone only by $t$ test (not shown) but not by analysis of covariance (Table I $A$ ), probably due to the skewed distribution of fiber occupancy in the older controls. While linear regression analysis in the combined nondiabetic controls revealed inverse linear relationships of myelinated fiber density and occupancy with age (Fig. 1, $\square$ and $O$ for IDDM and NIDDM controls, respectively, and dotted line for combined linear regression), adjustment of fiber density and occupancy for age by analysis of covariance only minimally altered the morphometric differences between the two diabetic groups and their respective age-matched controls (Table I $B$ ). Thus, fiber density and occupancy were markedly and similarly depressed in IDDM and NIDDM patients in a fashion that was independent of age. Fiber density and occupancy were also independent of both duration of diabetes and current hemoglobin $A_{1} C$ in NIDDM and IDDM patients. Because mean fascicular area was related to neither age nor diabetes, it is reasonable to conclude that the magnitude of sural nerve myelinated fiber loss was similar in IDDM and NIDDM patients with neuropathy, and that this loss largely obscured any agerelated change in fiber number.

Myelinated fiber size. $\chi^{2}$ analysis of the histographic size distribution of myelinated fibers (the "fiber caliber spectrum," Fig. 2) revealed statistically significant skewing toward smaller fibers in both IDDM and NIDDM patients vs. their respective age-matched controls (Fig. 2, $a$ and $b$ ), in IDDM patients vs. NIDDM patients (Fig. $2 c$ ), and in older NIDDM controls vs. IDDM controls (Fig. $2 d$ ). These spectral shifts accounted for the small and statistically insignificant reductions in mean fiber area in IDDM patients vs. controls, and in NIDDM controls vs. IDDM controls (Table I $A$, column 3 ). Linear regression analysis revealed corresponding inverse relationships between age and mean fiber size in both the combined controls (Fig. 3, $\bigcirc$ and $\square$, dotted line) and NIDDM patients $(\bullet$, dashed line) but not in IDDM patients ( $\square$ ) where fiber size tended to be displaced slightly downward independent of age. A corresponding trend was evident in the age-adjusted mean fiber size in the IDDM patients vs. the combined controls (Table $I B$, column 3) although this difference did not reach statistical significance. Thus subtle skewing toward smaller nerve fibers occurred with diabetic neuropathy and as a function of aging, with the more prominent shift exhibited by IDDM patients obscuring that associated with age.

A shift in the fiber caliber spectrum can reflect generalized changes in the size of existing fibers, or a change in the relative number of fibers within differently sized fiber subpopulations. Myelin sheath thickness and internodal length are both normally closely correlated with fiber size such that the natural logarithm of the axonal cross-sectional area is linearly related to the number of lamellae in the surrounding myelin sheath $(51,55,56)$, and fiber diameter is proportional to internodal length $(57,58)$. The number of myelin lamellae and the internodal length thus serve as normative markers for fiber crosssectional area, and changes in fiber cross-sectional area are detected as shifts in the slopes of these regression ratios (with fiber shrinkage decreasing the "axon-myelin" ratio and increasing the internodal length-fiber diameter ratio).

The slope of the axon-myelin ratio varied insignificantly

Table I A. Morphometric Characteristics of Sural Nerves in IDDM and NIDDM Patients and Age-matched Controls

\begin{tabular}{|c|c|c|c|c|c|c|}
\hline Groups & $(n)$ & Fiber density & Fiber occupancy & Mean fiber area & $\mathrm{CV}$ & Axo-glial dysjunction \\
\hline & & $m m^{-2}$ & $\%$ & $\mu m^{2}$ & $\%$ & $\%$ \\
\hline IDDM patients & (11) & {$\left[\begin{array}{l}3,198 \\
(1,244-4,768) \\
P<0.001\end{array}\right.$} & {$\left[\begin{array}{l}11.7 \\
(3.6-20.3) \\
P<0.001\end{array}\right.$} & $\begin{array}{l}36.2 \\
(28.6-51.0)\end{array}$ & $\begin{array}{l}13.3 \\
(5.6-28.7)\end{array}$ & {$\left[\begin{array}{l}35.3 \\
(18.4-43.3) \\
P<0.001\end{array}\right.$} \\
\hline IDDM controls & (8) & $\begin{array}{l}\lfloor 7,325 \\
\quad(5,806-9,144)\end{array}$ & $\begin{array}{l}\text { L30.4 } \\
\quad(25.5-37.9)\end{array}$ & $\begin{array}{l}42.8 \\
(31.2-52.0)\end{array}$ & $\begin{array}{l}14.2 \\
(7.9-32.8)\end{array}$ & $\begin{array}{l}\quad 7.5 \\
(3.8-12.4)\end{array}$ \\
\hline NIDDM patients & (17) & {$\left[\begin{array}{c}2,930^{*} \\
(754-6,009) \\
P<0.001\end{array}\right.$} & {$\left[\begin{array}{l}11.2^{*} \\
(1.5-21.3) \\
P<0.001\end{array}\right.$} & $\begin{array}{l}37.6 \\
(20.4-49.5)\end{array}$ & {$\left[\begin{array}{l}22.7^{\text {11 }} \\
(9.0-40.5) \\
P<0.025\end{array}\right.$} & $\begin{array}{l}17.6^{\ddagger * *} \\
(8.9-29.0)\end{array}$ \\
\hline NIDDM controls & (11) & $\begin{array}{l}\text { L6,546 } \\
\quad(3,970-9,318)\end{array}$ & $\begin{array}{l}{\left[20.2^{\ddagger}\right.} \\
\quad(2.9-35.8)\end{array}$ & $\begin{array}{l}35.5^{\S} \\
(28.7-51.5)\end{array}$ & $\begin{array}{l}L_{17.4} \\
(8.2-32.8)\end{array}$ & $\begin{array}{l}15.0^{\ddagger \S} \\
(5.9-28.6)\end{array}$ \\
\hline
\end{tabular}

Diagnostic fascicular sural nerve biopsies from neuropathic patients with IDDM or NIDDM entering an aldose reductase inhibitor trial were compared to sural nerve biopsies obtained from age-matched controls. Myelinated fibers were measured and counted from fixed and embedded sural nerve cross sections using computer-assisted light-microscopic imaging techniques. Fiber density (number of fibers per square millimeter) and fiber occupancy (percentage of cross-sectional area occupied by myelinated fibers) were computed and used as an estimate of myelinated fiber number. Mean myelinated fiber size was computed from fiber population histograms. CV of fiber density was computed by dividing the fascicular cross section into five radial segments, measuring the fiber density in each segment, and then computing the CV based on the five segments; this determination has been used to estimate the extent of nonhomogeneous (i.e., "focal") nerve fiber loss especially in ischemic neuropathies. Axo-glial dysjunction refers to the percentage of terminal loops of myelin that have lost the junctional complexes that normally anchor them to the paranodal axolemma (Fig. 7). Data are displayed as estimated means (and observed ranges) with $P$ values derived from ANOVA. ${ }^{*} P<0.001$ vs. IDDM controls. ${ }^{\ddagger} P<0.001$ vs. IDDM patients. ${ }^{\S} P<0.050$ vs. IDDM controls. $" P<0.005$ vs. IDDM patients. ' $P<0.025$ vs. IDDM controls. ${ }^{* *} P<0.005$ vs. IDDM controls. 

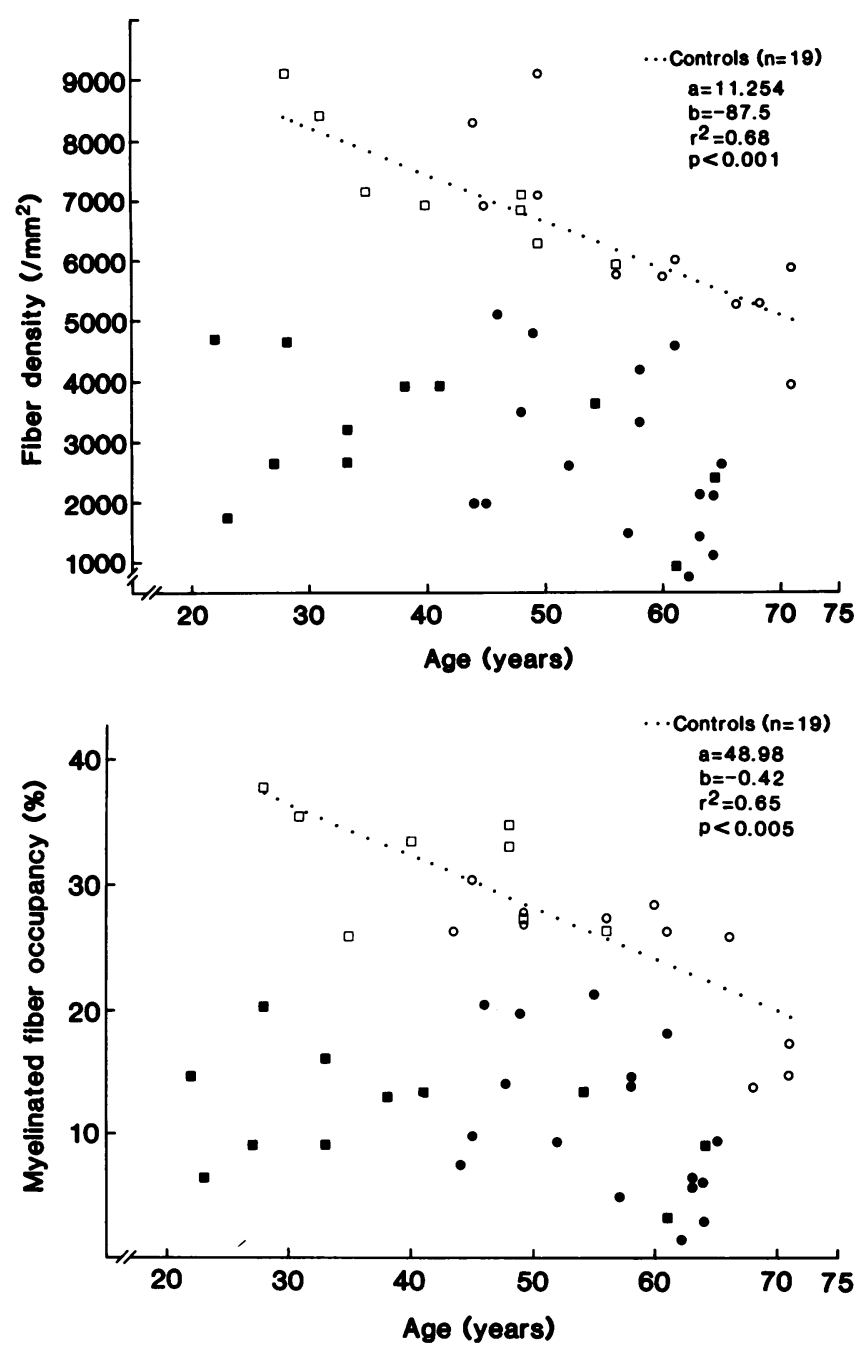

Figure 1. Effect of IDDM, NIDDM, and age on myelinated fiber density (upper panel) and occupancy (lower panel) in sural nerve biopsies. Myelinated fiber density (the number of myelinated fibers per square millimeter of cross-sectional area) and myelinated fiber occupancy (the percent cross-sectional area occupied by myelinated fibers) were computed as an estimate of myelinated fiber number in fascicular sural nerve biopsies obtained from neuropathy patients with IDDM ( $($ ) and NIDDM (•), and from nondiabetic control subjects age-matched to the IDDM or NIDDM patients ( $\square$ and o, respectively). These values were plotted as a function of the age of the subject, and linear regression analysis, indicated by the dotted line for the combined controls, was performed by the method of least squares. $n$, number of subjects; $a$ and $b$, intercept and slope of the regression line, respectively; and $r$, coefficient of correlation. No significant effect of age on fiber density and occupancy was observed in the NIDDM and IDDM patients. Analysis of covariance revealed that the shift toward lower fiber density and occupancy in diabetic patients compared with controls was highly statistically significant (Tables I $A$ and I $B$ ).

between 0.018 and 0.020 among the IDDM controls and the NIDDM patients and controls, but was significantly reduced to 0.012 in the IDDM patients (Fig. 4, upper panel). This reduction primarily reflected a decrease in the size of axons with thick myelin sheaths, although a simultaneous small increase in the size of axons with thin myelin sheaths suggested some accompanying regeneration and remyelination (Fig. 4, upper panel) (the presence of which is also supported by teased fiber analysis: see below). The slope of the internodal lengthfiber diameter ratio was increased $21 \%$ in the older NIDDM controls compared with the younger IDDM controls (Fig. 4, lower panel), implying that fiber diameter decreases slightly with age. The ratio was increased $69 \%$ in IDDM patients vs. only $19 \%$ in NIDDM patients compared to their respective age-matched controls (Fig. 4, lower panel). Taken together, these observations imply that selective loss of larger fibers may account for most of the normal age-related reduction in fiber size, whereas diabetic neuropathy, particularly that complicating IDDM, reduces the caliber of existing axons in a fashion analogous to that in animal models for IDDM.

Spatial distribution of myelinated fibers. Loss of large or small myelinated nerve fibers may occur in either a spatially uniform pattern or a focal pattern that varies within or among fascicles. Intra- or interfascicular focal fiber loss from putative areas of marginal vascular supply has been ascribed to "ischemic" injury whereas diffuse loss generally connotes "metabolic" insult $(1,50,52)$. The intra- and interfascicular focality of fiber loss has been assessed as the CV of fiber density (51). Intrafascicular CV was significantly increased in the NIDDM patients but not in the IDDM patients compared to their respective age-matched controls (Table I $A$, column 4, compare lines 1 and 2 with 3 and 4). Linear regression analysis confirmed a positive linear relationship between age and intrafascicular CV in the combined controls (Fig. 5, $\square$ and $\mathrm{O}$, dotted line) and IDDM patients (Fig. 5, a, solid line) but not in NIDDM patients (Fig. $5, \bullet$ ). Adjustment for age by analysis of covariance demonstrated significantly higher intrafascicular CV in NIDDM but not IDDM patients compared with combined controls (Table I $B$, column 4), and in NIDDM patients compared with IDDM patients (Table I $B$, column 4 , lines 1 and 3). When corrected for age, intrafascicular CV strongly and positively correlated with duration of diabetes in NIDDM patients (Fig. 6,, , dashed line) but not in IDDM patients (Fig. 6 , $\square$ ); it was unrelated to hemoglobin $A_{1} C$. Hence, increased focality of fiber loss was confined to NIDDM patients with neuropathy, could not be accounted for by age, and was positively related to duration but not severity of hyperglycemia.

Axo-glial dysjunction. Terminal loops of myelin in the paranodal region (Fig. 7) are tightly anchored to the paranodal axolemma by a collar of highly specialized junctional complexes that divide both the axolemma and the periaxolemmal extracellular space into nodal and internodal domains (Fig. 7, $d$ and $f$, arrowheads) (43). Axo-glial dysjunction signifies the loss of these strategic junctional complexes (41) (Fig. 7, $b$ and $d$, arrows). Axo-glial dysjunction correlates closely with antecedent paranodal swelling (40), and poorly reversible slowing of nerve conduction in the spontaneously diabetic BB rat (41), but has not been reported in human diabetic neuropathy.

Axo-glial dysjunction was increased approximately fivefold in IDDM patients but not in NIDDM patients compared to their respective age-matched controls (Table I $A$, column 5). Linear regression analysis revealed a positive linear relationship between age and axo-glial dysjunction in the combined controls (Fig. 8, $\square$ and O, dotted line) and the NIDDM patients (Fig. 8, $\bullet$, dashed line), with no statistically significant difference in axo-glial dysjunction between the latter two (Table I $B$, column 5). When corrected for age, axo-glial dysjunction correlated closely with duration of diabetes in IDDM (Fig. 9, $\mathbf{n}$, solid line) but not in NIDDM patients (Fig. 9, ๑). However, if one patient with NIDDM of $7 \mathrm{yr}$ in duration and an excep- 
Table I B. Age-adjusted Morphometric Characteristics of Sural Nerves in IDDM and NIDDM Patients and Age-matched Controls by Analysis of Covariance with Age Removed as a Covariant

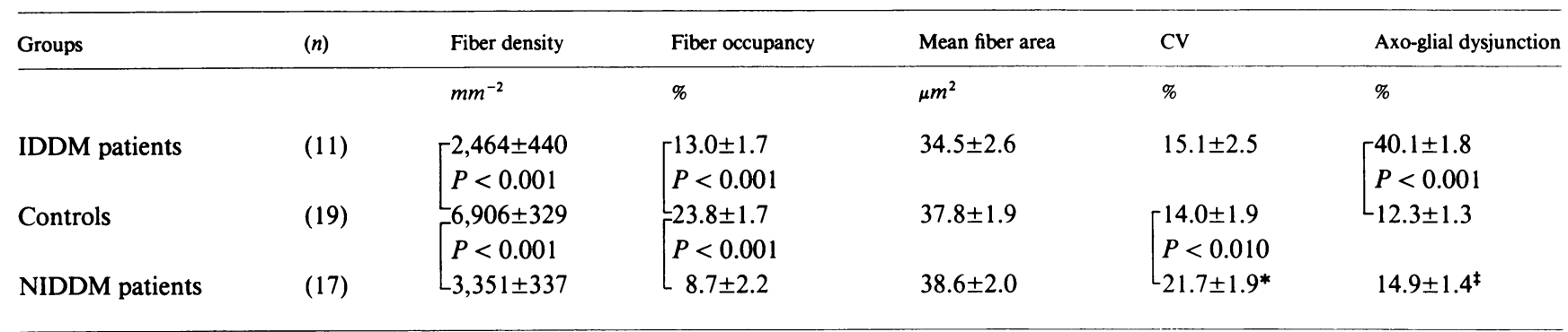

Data from Table I $A$ on IDDM and NIDDM patients and the combined controls are displayed as age-adjusted means \pm SEM computed by analysis of covariance with age eliminated as an independent covariant. The younger IDDM and older NIDDM controls were combined since values were corrected for differences in age. $P$ values derived from analysis of covariance. ${ }^{*} P<0.050$ vs. IDDM patients, ${ }^{\ddagger} P<0.001$ vs. IDDM patients.

tionally high frequency of axo-glial dysjunction is excluded from the evaluation, remaining NIDDM patients exhibited a significant positive correlation between axo-glial dysjunction and duration of diabetes (Fig. 9, $\bullet, a=8.54, b=0.79, r^{2}$
$=0.35, P<0.025)$. Axo-glial dysjunction did not correlate with current hemoglobin $\mathrm{A}_{1} \mathrm{C}$ in either diabetic group (data not shown). Thus, although axo-glial dysjunction increased slightly as a function of age in both the nondiabetic controls
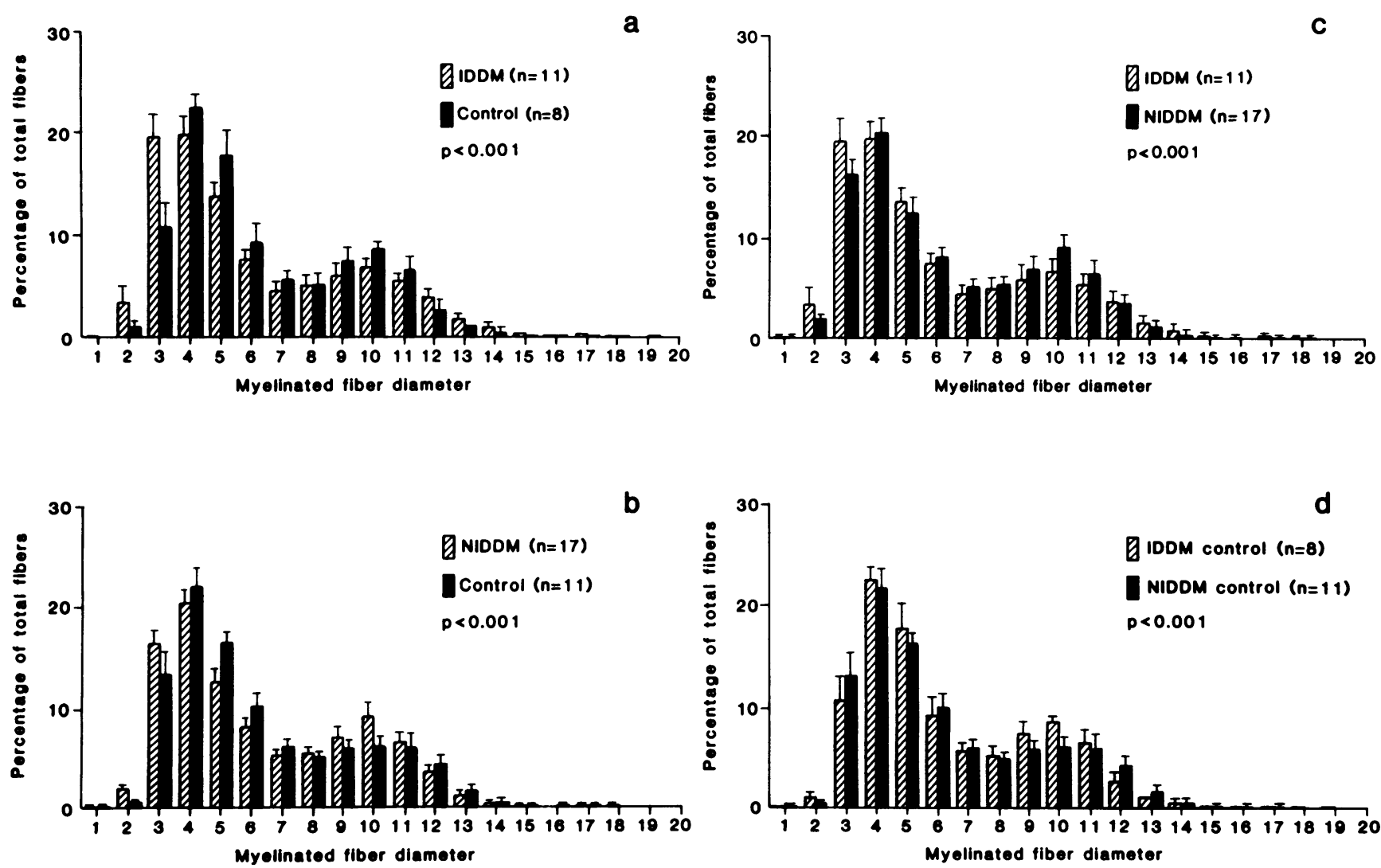

Figure 2. Effect of IDDM, NIDDM, and age on sural nerve myelinated fiber caliber spectrum. Histograms of size distribution of myelinated fibers for IDDM and NIDDM patients and age-matched controls were constructed using computer-assisted measurements of fiber areas from semithin toluidine blue-stained cross sections of sural nerve fascicles at a magnification of 1,600 . The height of each bar represents the mean percentage $( \pm S E M)$ of fibers within each increment in fiber size (in micrometers) plotted along the abscissa. The overall $P$ value for differences in fiber size distribution was calculated by $\chi^{2}$ analysis. Histograms from IDDM and NIDDM patients are

compared with those of their age-matched controls in $a$ and $b$. Histograms from IDDM patients and NIDDM patients are compared to each other in $c$. Histograms from the younger controls for the IDDM patients and the older controls from the NIDDM patients are compared in $d$. IDDM was associated with a clear shift of the fiber caliber spectrum toward smaller diameter fibers compared with agematched controls ( $a$ ), which was less evident in NIDDM patients $(b)$, as illustrated in $c$. Aging in the controls produced a similar skewing toward smaller nerve fibers as shown in $d$. 




Figure 3. Effect of IDDM, NIDDM, and age on mean myelinated fiber size. Mean fiber cross-sectional areas calculated from histograms like those in Fig. 2 were plotted as a function of age for each IDDM and NIDDM patient ( $\bullet$ and $\bullet$ ) and control ( $\square$ and $\circ$ ), respectively). Linear regression analysis revealed age-related decrements in mean fiber size in the combined controls and NIDDM patients (dotted and dashed lines) but not in IDDM patients. Mean fiber size was significantly reduced in the older NIDDM controls compared with the younger IDDM controls, but not in the IDDM or NIDDM patients (Tables I $A$ and I $B$, column 3). and the NIDDM patients (Fig. 8), it was markedly accentuated in patients with IDDM as a function of duration but not severity of hyperglycemia (Fig. 9).

Teased fiber analysis. Consecutively teased sural nerve fibers were morphologically categorized according to the criteria of Dyck and co-workers $(50,52)$ as modified for diabetic neuropathy on the basis of recent experience in the BB rat model (39-41). The percentage of teased fibers with normal appearance was markedly decreased in IDDM and NIDDM patients to $\sim 40 \%$ and $55 \%$ of that of their respective agematched controls (category A, Table II $A$, line 1). Neuropathy in IDDM and NIDDM patients correspondingly increased the frequency of most types of abnormal nerve fibers. The frequency of fibers exhibiting paranodal swelling (Fig. 10, panels 1 and 2; category B, Table II $A$, line 2) was increased 20- and 10-fold, respectively, in IDDM and NIDDM patients with neuropathy. Paranodal demyelination (Fig. 10, panels 3-5; category C, Table II $A$, line 3 ) was increased $\sim 40$ - and 6-fold in IDDM and NIDDM patients correspondingly increased the tercalated (remyelinated) nodes (Fig. 10, panel 6; category E, Table II $A$, line 5) were increased fourfold in IDDM but not NIDDM patients vs. age-matched controls, and were correlated with paranodal demyelination $(P<0.010$ by linear regression analysis); however, when corrected for age by analysis
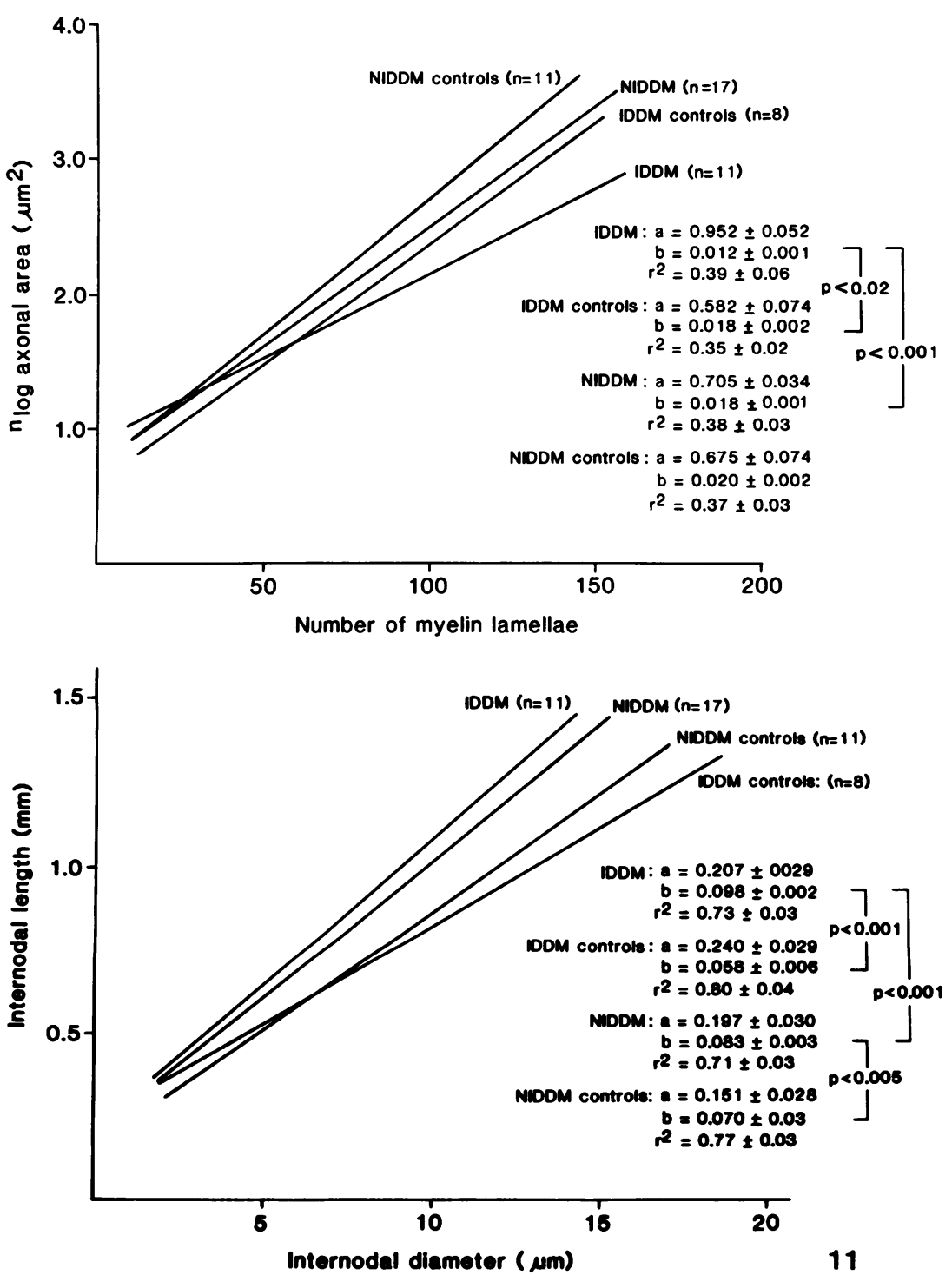

Figure 4. Effect of IDDM, NIDDM, and age on axon-myelin ratio (upper panel) and internodal length-diameter ratio (lower panel). For axonmyelin ratio, the natural logarithm of the axonal cross-sectional area and the number of surrounding myelin lamellae were measured on electron-microscopic prints and plotted as recommended by O'Neill Gilliatt (51) for $\sim 40$ randomly selected myelinated fibers from each fascicular biopsy. The slopes of the linear regressions generated from these points for each subject ( $b$ in the equation $y=a+b x$ ) were compared for IDDM and NIDDM patients and their respective age-matched controls. The intercept of the regression line for IDDM patients was significantly higher than the IDDM controls only at a myelin thickness of 10 lamellae $(P$ $<0.050$ ), and was significantly lower at myelin thicknesses of 100,125 , and 150 lamellae $(P$ $<0.050,<0.010,<0.010)$ indicating that the shift in slope was primarily due to a decrease in the axon-myelin ratio of large myelinated fibers rather than an increase in the ratio in small fibers (21). For internodal length-diameter ratio, the internodal length of teased fibers was measured using an eyepiece caliper, and internodal diameter measured at five equidistant points along the internode. The internodal length and the mean of the five measurements of fiber diameter were plotted, and compared by linear regression analysis as above for IDDM and NIDDM patients and their respective agematched controls. Reduction in the axon-myelin ratio or increase in the internodal lengthdiameter ratio generally connotes loss of axonal cross-sectional area. IDDM patients had the lowest axon-myelin and the highest internodal length-diameter ratios, consistent with the most prominent axonal shrinkage. 


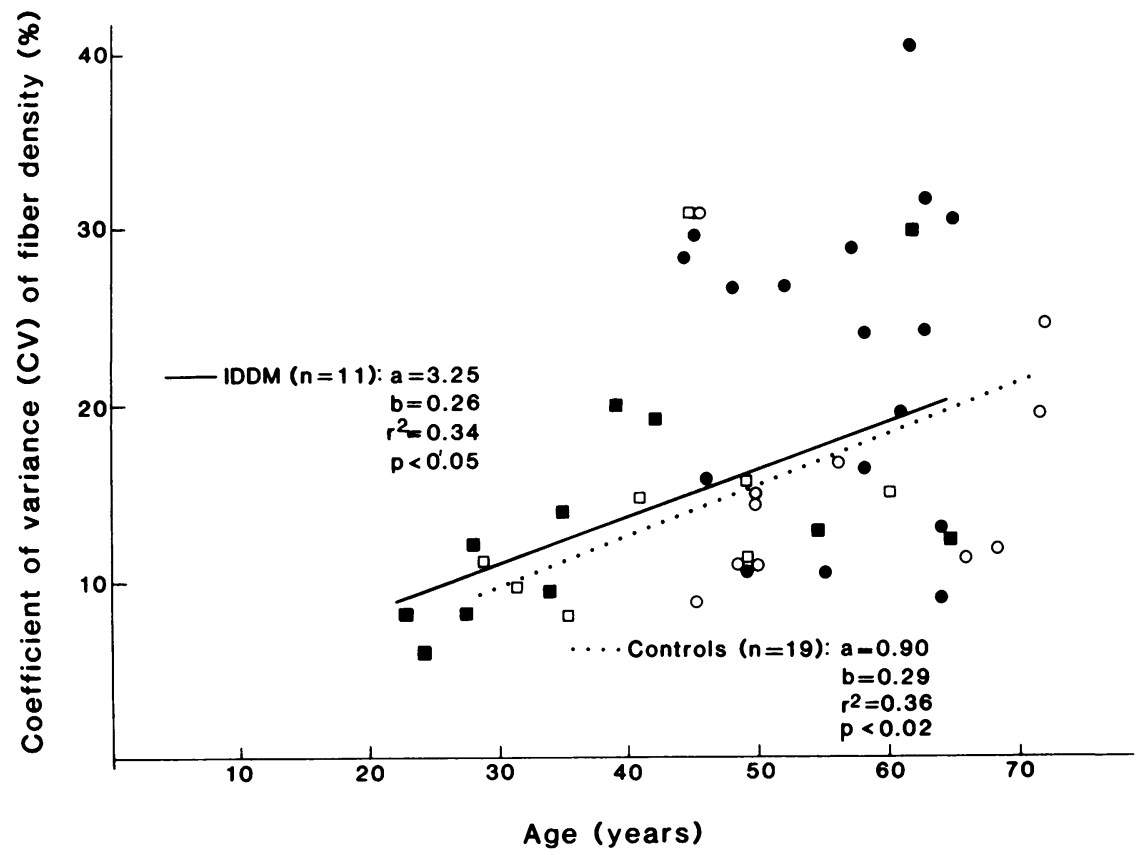

Figure 5. Effect of IDDM, NIDDM, and age on $\mathrm{CV}$. The variation of fiber density among radial sectors of each fascicular biopsy was determined as described in Methods and plotted against the age of each IDDM and NIDDM patient ( $\square$ and $\bullet$, respectively) and their respective age-matched controls ( $\square$ and o). Linear regression analysis revealed a positive correlation between age and $\mathrm{CV}$ in the combined controls and the IDDM patients, but not in the NIDDM patients in whom CV correlated with duration of diabetes (Fig. 6). CV was significantly increased in the NIDDM but not the IDDM patients compared with their respective age-matched controls (Table I $A$, column 4) or compared with the combined controls after correction for differences in age (Table I $B$, column 4).

of covariance and compared with the combined controls, the frequency of intercalated nodes was increased in both IDDM and NIDDM patients (category E, Table II $B$, line 5). Segmental demyelination (category $\mathrm{G}$, Table II $A$, line 7) was increased nine- and fivefold in IDDM and NIDDM patients, respectively. Myelin wrinkling (Fig. 11; category D, Table II $A$, line 4), conventionally interpreted as a secondary response to axonal shrinkage, was increased 13- and 6-fold in IDDM and NIDDM patients, respectively, compared with their agematched controls. (Linear regression analysis of age-corrected values confirmed the generally accepted association of excess myelin wrinkling with other hallmarks of axonal shrinkage, i.e., decreased axon-myelin ratio $[P<0.001$ in combined controls and IDDM patients and $P<0.010$ in NIDDM patients] and increased internodal length-fiber diameter ratio $[P$
$<0.025$ in combined controls, $P<0.005$ in IDDM patients and $P<0.050$ in NIDDM patients]). Wallerian degeneration (category $\mathrm{F}$, Table II $A$, line 6) was increased about twofold in both IDDM and NIDDM patients. Fibers exhibiting regeneration and/or remyelination were increased fourfold in both IDDM and NIDDM patients compared with their respective age-matched controls (category $\mathrm{H}$, Table II $A$, line 8).

Thus, the abnormalities at the node of Ranvier exhibited by teased nerve fibers from patients with IDDM and NIDDM were qualitatively similar and included paranodal swelling, demyelination, and remyelination. The overall frequency of nodal pathology was fourfold greater in the IDDM patients than in NIDDM patients compared to their respective agematched controls (Table II $A$, sum of categories $\mathrm{B}, \mathrm{C}$, and $\mathrm{E}$ ), but this statistically insignificant quantitative difference was

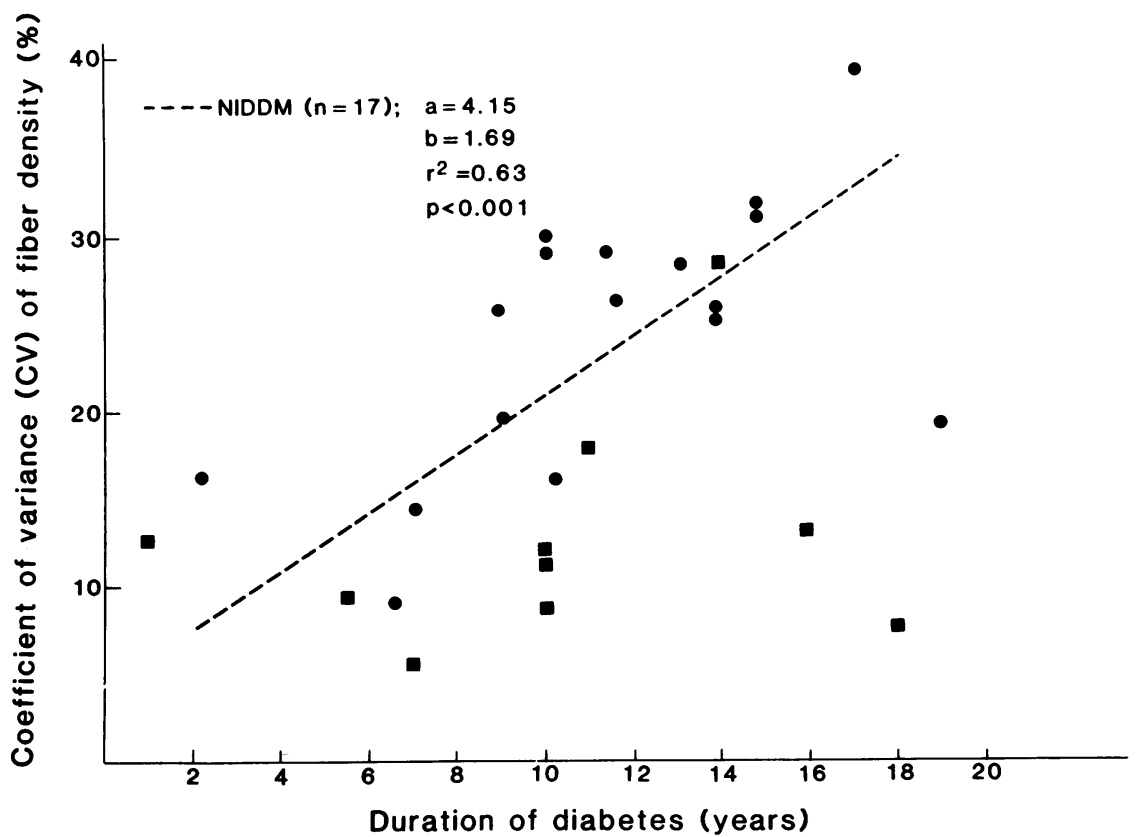

Figure 6. Effect of duration of IDDM and NIDDM on CV. The values for CV in the IDDM patients ( $(\bullet)$ and NIDDM patients ( $\bullet$ ) were plotted against duration of diabetes and analyzed by linear regression. The abnormally increased CV values in NIDDM patients were linearly related to duration of diabetes, whereas the normal $\mathrm{CV}$ values in IDDM patients were not related to duration of diabetes. 

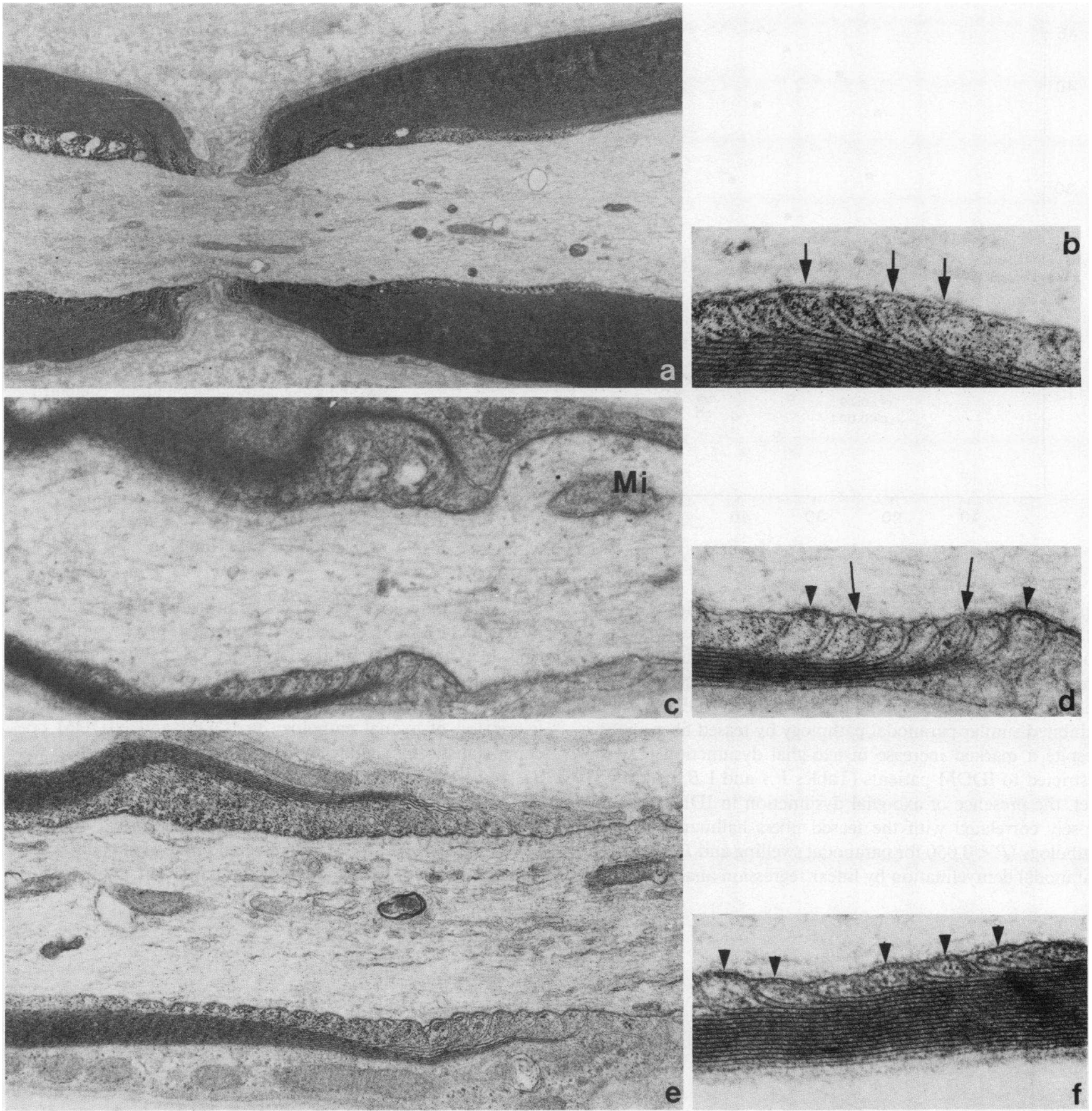

Figure 7. Axo-glial dysjunction and axonal swelling in a fascicular nerve biopsy from a patient with neuropathy complicating IDDM. (a) Longitudinal section through the nodal and paranodal areas of a large myelinated axon with apparent normal architecture and normal abutment of the terminal myelin loops. (b) Higher magnification of the right lower portion of the micrograph shows terminal myelin loops which are well apposed to the axolemma but without axoglial junctional complexes (arrows). Myelinated fibers showing a high frequency of axo-glial dysjunction seldom displayed nodal and paranodal swelling. (c) This longitudinal section of the nodal and paranodal area shows distortion of the nodal architecture and axonal swelling of the node. Mi, mitochondrion. This abnormality was usually not associated with the absence of axo-glial junctional complexes ("axo-glial dysjunction"). (d) In this particular fiber, however, axo-glial dysjunction was noted (arrows). Preserved axo-glial junctions are also visible as electron-dense structures between the axolemma and the plasma membrane surrounding the terminal loops (arrowheads). In IDDM patients a highly significant $(P<0.001)$ association was found between the frequency of paranodal swelling and that of axo-glial dysjunction suggesting a causal relationship between these two abnormalities. (e) Longitudinal section through the nodal and paranodal area of a myelinated fiber of a control nerve. No nodal swelling is seen and the terminal myelin loops are closely apposed to the axolemma. $(f)$ As demonstrated in the high-magnification micrograph, the myelin loops are anchored to the axolemma by electron-dense axo-glial junctional complexes (arrowheads). Magnifcations: $(a) \times 4,194 ;(b) \times 62,100 ;(c) \times 13,500 ;(d) \times 55,080 ;(e)$ $\times 10,620 ;(f) \times 55,080$. 


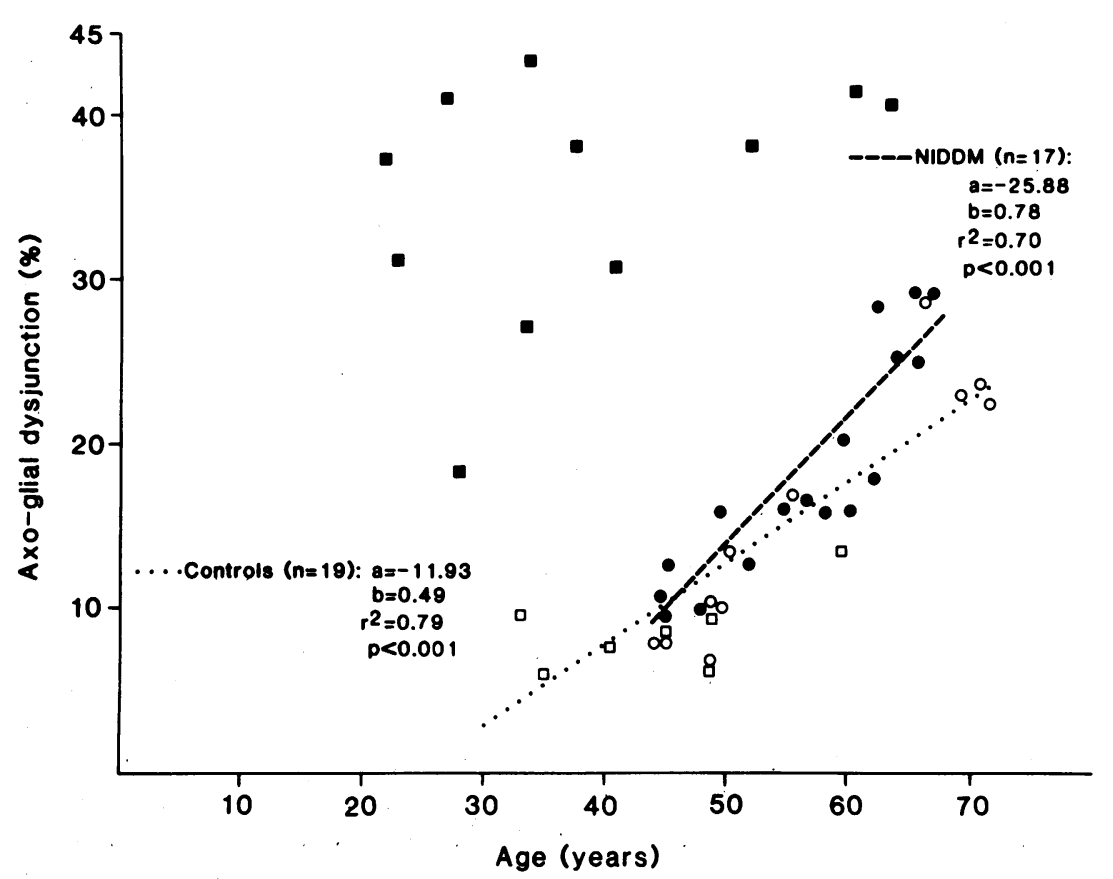

Figure 8. Effect of IDDM, NIDDM, and age on axo-glial dysjunction. The number of terminal myelin loops without discernible axo-glial junction complexes (see Fig. 7) was determined in nearly 400 loops per fascicular biopsy and expressed as a percentage. This percentage of axoglial dysjunction was plotted as a function of the age of the subject for IDDM and NIDDM subjects ( $\bullet$ and $\bullet$, respectively) and their agematched controls (open symbols). Regression analysis revealed linear relationships between age and axo-glial dysjunction for the combined controls and the NIDDM patients, but not for the IDDM patients in whom axo-glial dysjunction correlated with duration of diabetes (Fig. 9). Axo-glial dysjunction was increased signifcantly in the IDDM but not the NIDDM patients compared to their respective controls (Tables I $A$ and I $B$, column 5). eliminated by age correction (Table II $B$, categories B, C, and E). This is in stark contrast to the marked increase in paranodal axo-glial dysjunction in IDDM patients. (Table I $A$, column 5) that persists following correction for age (Table I $B$, column 5). Thus, neuropathy in IDDM and NIDDM patients exhibited similar paranodal pathology by teased fiber analysis despite a marked increase in axo-glial dysjunction that was restricted to IDDM patients (Tables I $A$ and I $B$, column 5). Yet, the presence of axo-glial dysjunction in IDDM patients closely correlated with the teased fibers hallmarks of nodal pathology $(P<0.050$ for paranodal swelling and $P<0.025$ for paranodal demyelination by linear regression analysis).
Axonal pathology was expressed in teased fibers primarily by a tendency for an exaggerated increase in myelin wrinkling (Fig. 11; Tables II $A$ and II $B$, category D) in IDDM patients, and for increased Wallerian degeneration (Tables II $A$ and II $B$, category F) in NIDDM patients. This tendency for alternative expression of axonal pathology in IDDM and NIDDM became statistically significant when values were corrected for age and compared to the combined controls (Table II $B$, categories $\mathrm{d}$ and $\mathrm{f}$; compare columns 1 and 3 ). Increased myelin wrinkling in IDDM patients correlated with axo-glial dysjunction $(r=0.798, P<0.001)$, which could magnify myelin wrinkling by permitting paranodal myelin retraction. On the other

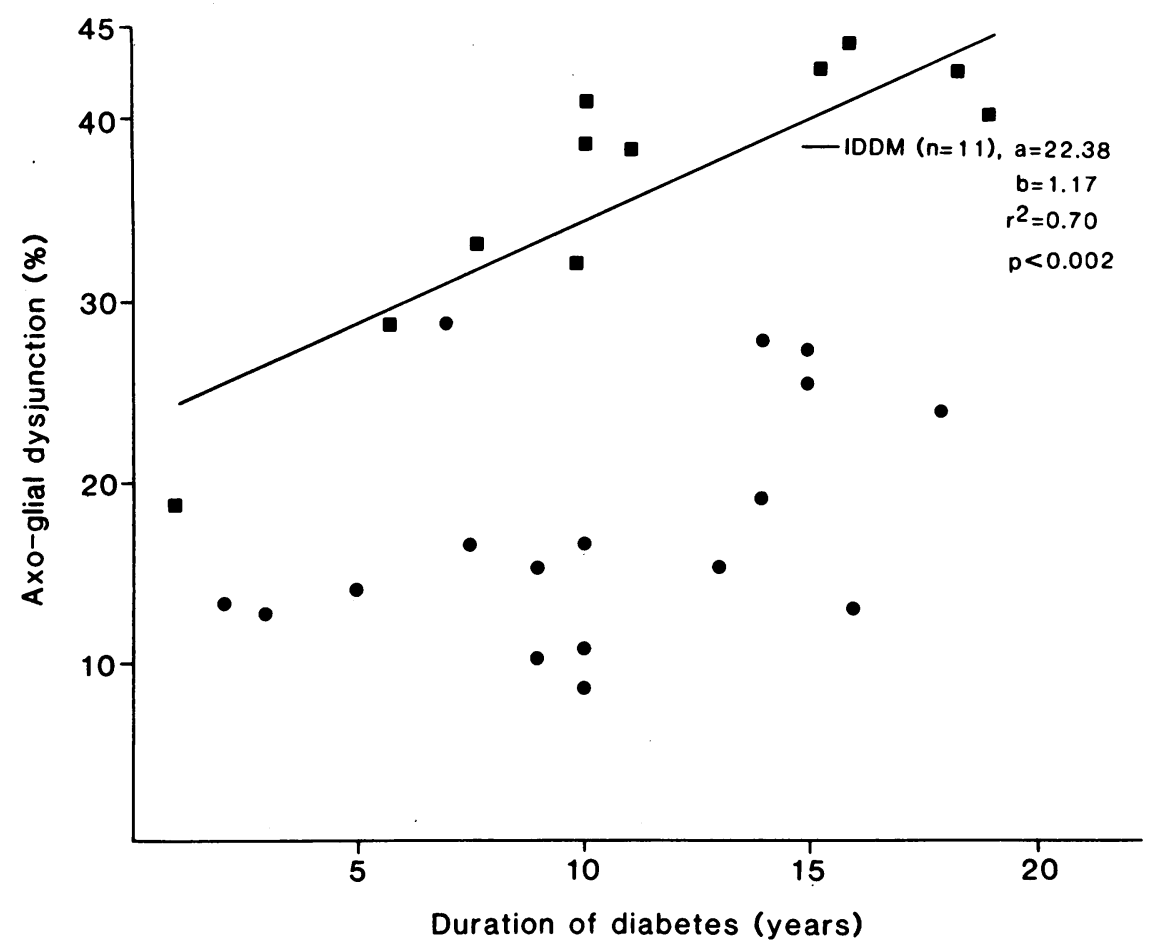

Figure 9. Effect of duration of diabetes on axo-glial dysjunction in IDDM and NIDDM patients. Axo-glial dysjunction increased linearly with duration of diabetes in IDDM ( $(\mathbf{)})$ but not NIDDM (•) patients in whom it was not increased over age-matched controls (Fig. 7) and Tables I $A$ and $I B$ ). 
Table II A. Scored Pathology of Teased Fibers in Sural Nerve Biopsies from IDDM and NIDDM Patients and Age-matched Controls

\begin{tabular}{|c|c|c|c|c|}
\hline Fiber categories & \multicolumn{2}{|l|}{ IDDM } & \multicolumn{2}{|l|}{ NIDDM } \\
\hline \multirow{2}{*}{ A. Normal fibers } & $37.5(25.5-48.0)$ & $90.6(79.1-94.8)$ & $45.8(17.2-70.6)^{*}$ & $82.3(64.2-92.8)^{\ddagger}$ \\
\hline & \multicolumn{2}{|c|}{$P<0.001$} & \multicolumn{2}{|c|}{$P<0.001$} \\
\hline \multirow[t]{2}{*}{ C. Paranodal demyelination } & $11.4(7.0-16.4)$ & $0.3(0.00-0.70)$ & $11.1(5.3-17.5)^{*}$ & $2.0(0.30-5.8)^{\ddagger}$ \\
\hline & \multicolumn{2}{|c|}{$P<0.001$} & \multicolumn{2}{|c|}{$P<0.001$} \\
\hline \multirow[t]{2}{*}{ D. Myelin wrinkling } & $26.0(13.9-36.0)$ & $2.0(0.0-5.4)$ & $17.8(3.9-34.9)^{*}$ & $3.0(0.3-6.5)^{\ddagger}$ \\
\hline & \multicolumn{2}{|c|}{$P<0.001$} & \multicolumn{2}{|c|}{$P<0.001$} \\
\hline E. Intercalated nodes & $5.2(1.3-11.0)$ & $1.2(0.0-3.4)$ & $5.9(2.0-10.1)^{*}$ & $4.1(0.7-9.6)$ \\
\hline \multirow[t]{2}{*}{ G. Segmental demyelination } & $4.5(1.6-8.8)$ & $0.5(0.0-1.3)$ & $5.3(0.5-12.8)^{*}$ & $1.1(0.0-2.5)^{8}$ \\
\hline & \multicolumn{2}{|c|}{$P<0.005$} & \multicolumn{2}{|c|}{$P<0.001$} \\
\hline \multirow[t]{2}{*}{ H. Regeneration/remyelination } & $8.0(1.7-16.1)$ & $1.9(0.2-3.9)$ & $6.2(1.0-15.1)^{11}$ & $1.4(0.1-3.9)^{\ddagger}$ \\
\hline & \multicolumn{2}{|c|}{$P<0.001$} & \multicolumn{2}{|c|}{$P<0.001$} \\
\hline
\end{tabular}

Individual myelinated fibers were teased from fascicular sural nerve biopsies obtained from neuropathic patients with IDDM and NIDDM and age-matched controls, and categorized based on morphological criteria modified from (53) as described in detail (Sima et al., manuscript in preparation) and observed by phase-contrast microscopy. Data are displayed as estimated means (and observed ranges) with $P$ values derived from an ANOVA. ${ }^{*} P<0.001$ vs. IDDM controls. ${ }^{\ddagger} P<0.001$ vs. IDDM patients. ${ }^{\S} P<0.005$ vs. IDDM patients. $\| P<0.005$ vs. IDDM controls.

Table II B. Age-adjusted Scored Pathology of Teased Fibers in Sural Nerve Biopsies Obtained from IDDM and NIDDM Patients and Combined Controls



Data from Table II $A$ on IDDM and NIDDM patients and combined controls are displayed as age-adjusted means \pm SEM computed by analysis of covariance with age eliminated as an independent covariant. All categories of teased fiber pathology except categories $\mathrm{C}$ and $\mathrm{E}$ showed significant correlations with age in the controls. $P$ values were derived from analysis of covariance. $* P<0.005$ vs. IDDM patients. ${ }^{\ddagger} P<0.001$ vs. IDDM patients. ${ }^{\delta} P<0.050$ vs. IDDM patients. 


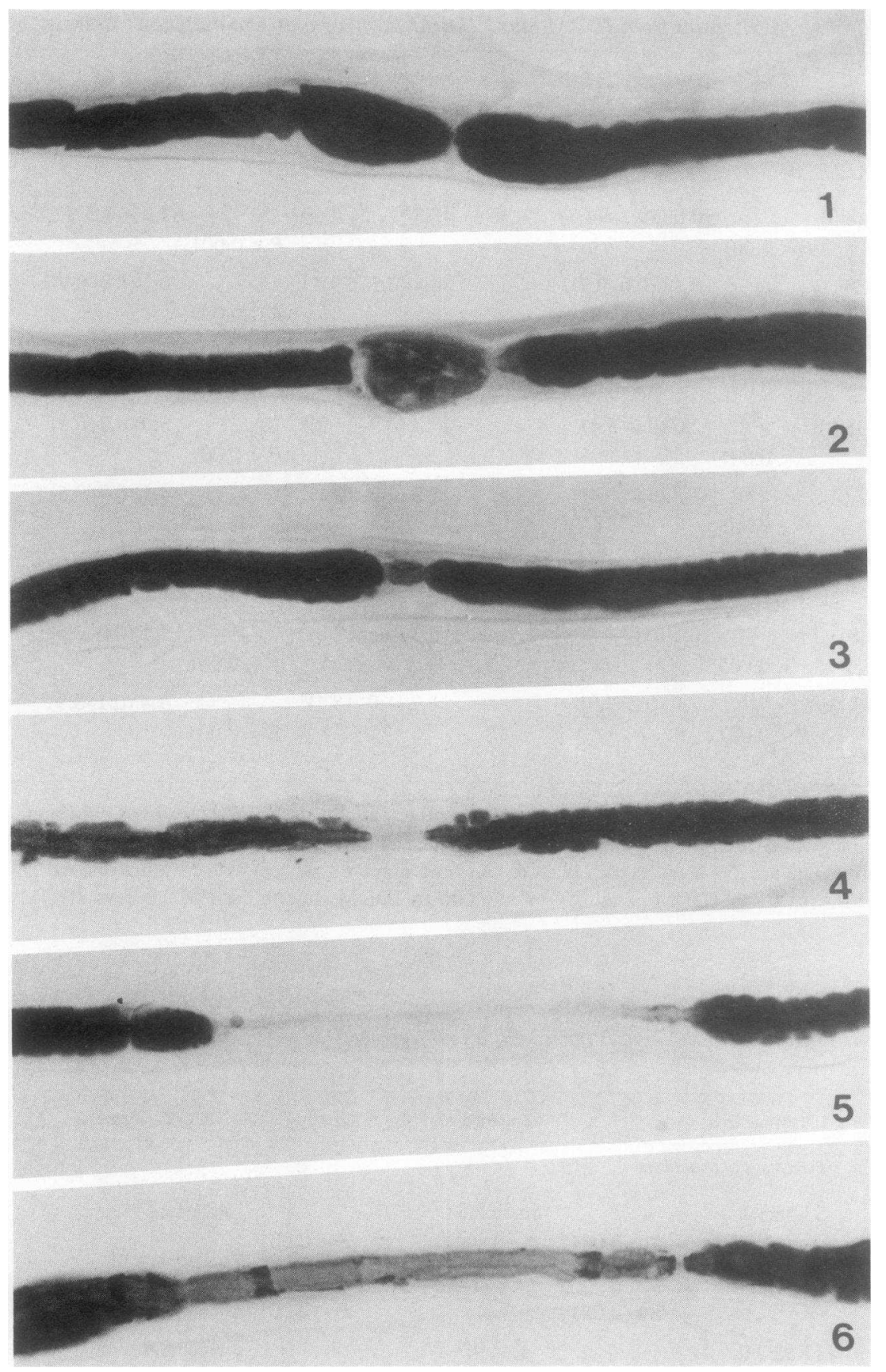

Figure 10. The effect of diabetes on the paranodal apparatus of myelinated fibers reconstructed in sequence. A series of teased fibers from patients with IDDM were arranged to illustrate the presumed sequence of nodal structural change beginning with paranodal swelling (panels 1 and 2; category B, Tables II $A$ and II $B)$, progressing through paranodal demyelination with myelin retraction (panels 3-5; category C, Tables II $A$ and II $B$ ) to paranodal remyelination (intercalated node) (panel 6; category E, Tables II $A$ and II $B$ ). The nerve fibers were osmicated and teased in Epon as described in Methods. $\times 720$. hand, axo-glial dysjunction and Wallerian degeneration were inversely correlated in IDDM patients $(r=-0.716, P$ $<0.005$ ). Thus, axo-glial dysjunction and associated axonal atrophy and myelin wrinkling were distinctive characteristics of fiber damage in IDDM patients, whereas Wallerian degeneration accompanied the more focal fiber loss in NIDDM patients.

\section{Discussion}

The distal symmetric form of diabetic neuropathy in both humans and animals is characterized by a spatially heterogeneous degeneration and loss of distal portions of large and small myelinated nerve fibers $(1,15,20,29,40,44,59-62)$ accompanied by endoneurial vascular abnormalities $(1,17$,
20) and associated with proximal focal lesions resembling those attributed to nondiabetic vascular insufficiency $(1,20$, 21). Myelinated fiber density and occupancy (measures of fiber number) and mean myelinated fiber cross-sectional area (a measure of fiber size) decreased as a function of age in a nondiabetic control population, most likely reflecting a selective loss of large myelinated nerve fibers. Fiber loss in control subjects coincided with an increase in the intrafascicular sectorial CV of fiber density, which, by the criteria of Dyck and co-workers $(50,52)$ would imply an age-dependent relative ischemia of peripheral nerve in nondiabetic subjects. In order to distinguish the similar effects of age and diabetes on sural nerve morphometry and teased fiber analysis, age matched controls were considered mandatory for proper interpretation of diabetic nerve biopsies. 

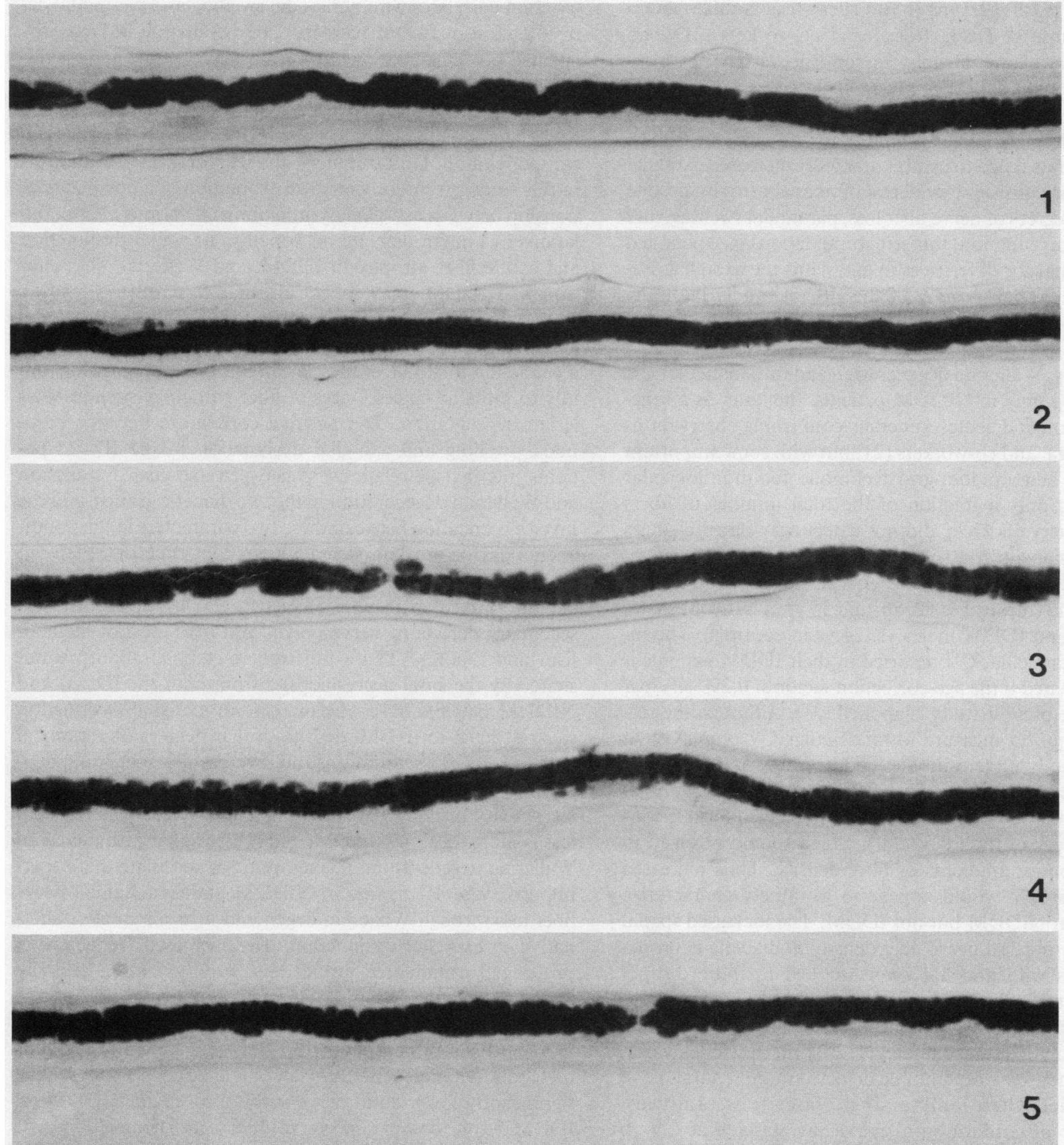

Figure 11. Excessive myelin wrinkling of a single teased nerve fiber. Excessively wrinkled myelin was the most frequently observed abnormality in teased sural nerve fibers from both IDDM and NIDDM patients (Table I $A$, line 4, category D). It correlated with the morphometric hallmarks of axonal atrophy such as the axon-myelin and

Therefore, morphometric data from sural nerve biopsies obtained from younger IDDM and older NIDDM patients with neuropathy were compared with strictly age-matched controls and subjected to correction for age differences by analysis of covariance. Myelinated fiber density and occupancy were comparably diminished in IDDM and NIDDM patients compared with their respective age-matched controls. Paranodal pathology (paranodal swelling, demyelination, and remyelination), myelin wrinkling, segmental demyelination, and fiber regeneration and remyelination were increased in biopsies from both IDDM and NIDDM patients compared internodal length-diameter ratios (Fig. 4) and with axo-glial dysjunction (Fig. 7). Its greater prevalence in IDDM vs. NIDDM patients (Table II $B$, line 4 , category D) may reflect the greater axonal atrophy alone, or the additive effects of atrophy plus axo-glial dysjunction in IDDM. $\times 410$.

with age-matched or age-corrected controls, as has been noted by others $(20,21,25,28,34)$.

Detailed structural analysis of the node of Ranvier in sural nerve biopsies from IDDM patients revealed the entire array of statistically interrelated changes described in the BB rat model (2) including paranodal swelling, axo-glial dysjunction (39-41), and subtle axonal atrophy (44) (the last of which is found also in other animal models for IDDM such as the streptozotocin diabetic rat, in which mean tibial nerve fiber size diminishes without a change in fiber number, and analysis of axon-myelin ratio ascribes this shift entirely to a reduction 
in the size of existing axons [63]). These results differ somewhat from those of Dyck, Rizza, and co-workers (21) who failed to detect axonal atrophy in sural nerve biopsies from patients with IDDM using only a single (nlog axon/myelin area) rather than multiple parameters for axonal atrophy, and employing a less favorable technique for its assessment (51). NIDDM patients failed to exhibit markedly increased axo-glial dysjunction or consistent evidence of axonal atrophy despite comparable degrees of nerve fiber loss, paranodal swelling, and paranodal demyelination; this structural discordance persisted after adjustment for differences in age. Thus the axo-glial dysjunction and axonal atrophy of the BB rat model faithfully simulate the neuropathology of IDDM, but less so that of NIDDM.

Conversely, Wallerian degeneration and intrafascicular CV were increased only in NIDDM patients, the latter as a function of duration of diabetes, generally confirming observations by Dyck, Rizza, and co-workers (21) but with some important differences. Their random-grid technique for intrafascicular $\mathrm{CV}$, counting only a fraction of the total number of fibers within each nerve fascicle, did not detect age-related changes in their nondiabetic controls and IDDM patients; therefore, they compared their major diabetic groups to controls less than half as old (mean ages 52, 56, and $55 \mathrm{yr}$ in NIDDM males and females and IDDM males vs. $24 \mathrm{yr}$ in controls). The increased intrafascicular CV reported in their IDDM patients is largely eliminated if the age-correction factor of $0.29 \% \mathrm{CV}$ per year from the present study is applied (21). Although the sectorial technique to measure intrafascicular CV employed in the present study underestimates concentric variation in fiber density, spatial variation in fiber density in diabetic neuropathy results from wedge-shaped or crescent-shaped focal lesions at the periphery of the fascicle (1), which should not lead to strictly concentric gradients of fiber density. Thus increased intrafascicular CV would appear to genuinely characterize neuropathy in NIDDM but not IDDM. The increased spatial heterogeneity in sural nerve fiber density in diabetic neuropathy correlates with and has been ascribed to more distinct intra- and interfascicular focal regions of fiber loss that have been detected at more proximal levels within the peripheral nervous system in autopsy studies $(1,20)$ (interfascicular variation in fiber density would not be detected in most sural nerve biopsy studies where only a single fascicle is sampled). Whether the discordant sural nerve intrafascicular $\mathrm{CV}$ in IDDM and NIDDM reflect corresponding differences in proximal nerve pathology, or divergent peripheral responses to similar degrees of proximal inter- or intrafascicular focal damage, remains to be established.

This morphological discordance in sural nerve biopsies from patients with neuropathy complicating IDDM and NIDDM suggest a number of possible temporal and/or sequential differences in the underlying pathogenesis. Distal symmetric polyneuropathy in IDDM and NIDDM is unlikely to reflect entirely and fundamentally different pathogenetic processes because of the many shared clinical, biochemical, electrophysiological, and morphological characteristics. Axoglial dysjunction and fiber atrophy might comprise epiphenomena unrelated to the postulated sequence leading from paranodal swelling to more advanced alterations in nodal architecture such as paranodal demyelination; this is unlikely because myelin retraction would presumably entail disruption of axo-glial junctional complexes, and because axo-glial dys- junction correlates with paranodal swelling and paranodal demyelination in IDDM patients. The population of large myelinated nerve fibers shown to be most susceptible to diabetesinduced axo-glial dysjunction in the BB rat model (41) may have been depleted from the older NIDDM patients by the age-related selective fiber loss detected in the nondiabetic control population. Unfortunately, the small number of subjects in this study precluded statistical evaluation of a possible relationship between axo-glial dysjunction and various fiber populations in human diabetic neuropathy. Axo-glial dysjunction and subtle fiber atrophy in IDDM, and Wallerian degeneration in NIDDM, may reflect alternative pathways leading from the early metabolic damage (expressed as paranodal swelling) to ultimate loss of nerve fibers. For instance, axo-glial dysjunction might be evanescent in NIDDM, progressing rapidly to more advanced stages of fiber pathology such as Wallerian degeneration. The positive correlation between paranodal swelling and axo-glial dysjunction in the IDDM patients, the inverse relationship between axo-glial dysjunction and Wallerian degeneration in the two diabetic groups, and the inverse correlation between these two parameters in the nondiabetic controls are consistent with this construct. In the BB rat, paranodal swelling $(39,40)$ and probably axo-glial dysjunction (41) are linked to insulin deficiency and hyperglycemia via secondary defects in nerve polyol and myo-inositol metabolism and (Na,K)-ATPase function, metabolic abnormalities shared by the sural nerve biopsies from both the IDDM and NIDDM patients (64). The increase in axo-glial dysjunction was restricted to IDDM, despite similar degrees of paranodal swelling, fiber loss, duration, and severity of diabetes and neuropathy, and reduced myo-inositol content and (Na,K)-ATPase activity in both diabetic groups (64). Because nerve fiber loss is more focal in NIDDM, and is associated with increased Wallerian degeneration, it is tempting to speculate that superimposed vascular factors in NIDDM patients channel nerve fiber loss through Wallerian degeneration as a sequel or alternative to axo-glial dysjunction. The increased frequency of prolonged undetected antecedent hyperglycemia and the higher prevalence of symptomatic neuropathy at diagnosis in NIDDM patients (45) suggests that they may exhibit a more indolent form of neuropathy possibly reflecting a less serious metabolic derangement; this view would be consistent with the significantly lower sural nerve sorbitol levels in NIDDM vs. IDDM despite comparable nerve glucose and hemoglobin $\mathrm{A}_{1} \mathrm{C}$ levels (64). Prevention and/or treatment studies using specific metabolic intervention, plus additional detailed structural studies in diabetic and nondiabetic subjects will be required to definitively differentiate among these various possibilities.

These studies strongly support the relevance of the BB rat model and its linked sequential biochemical, biophysical, and morphological pathogenetic mechanisms $(2,9,42,44)$ to human diabetic neuropathy, especially in IDDM. Despite significant similarities, the pathogenetic picture of neuropathy in NIDDM appears slightly more complex than in IDDM, with structural characteristics compatible with a superimposed vascular component. This subtle pathogenetic variability should be recognized in the design, implementation, and interpretation of future clinical trials in diabetic polyneuropathy. Many of the same metabolic abnormalities involving sorbitol, myoinositol and $(\mathrm{Na}, \mathrm{K})$-ATPase that have been invoked in the primary fiber damage in the $\mathrm{BB}$ rat have now been identified in vascular elements $(6,7,13,14)$ including endoneurial capil- 
laries (7). The recent suggestion that $(\mathrm{Na}, \mathrm{K})$-ATPase inhibition produces an exaggerated vasoconstrictive response in neural vessels $(65)$ raises the intriguing possibility that metabolically induced abnormalities in vascular tone superimposed on macroangiopathy in NIDDM patients may become limiting for nerve fiber survival. Thus, similar defects in sorbitol, myo-inositol and (Na,K)-ATPase metabolism in the nodal region of large myelinated nerve fibers as well as in other cellular components of peripheral nerve may contribute to the pathogenesis of neuropathy in IDDM and NIDDM patients via subtly different routes depending upon conditioning factors such as age-related vascular disease.

\section{Acknowledgments}

The authors gratefully acknowledge the statistical advice provided by Dr. Morton Brown of the Biostatistics Core of the Michigan Diabetes Research and Training Center.

These studies were supported by a research grant from Pfizer Central Research Division of Pfizer Pharmaceuticals Inc., Groton, CT.

\section{References}

1. Johnson, P. C., S. C. Doll, D. W. Cromey. 1986. Pathogenesis of diabetic neuropathy. Ann. Neurol. 19:450-457.

2. Sima, A. A. F. 1985. Annotation: can the BB-rat help to unravel diabetic neuropathy? Neuropathol. Appl. Neurobiol. 11:253-264.

3. Greene, D. A., S. A. Lattimer, J. Ulbrecht, and P. Carroll. 1985. Glucose-induced alterations in nerve metabolism: current perspective on the pathogenesis of diabetic neuropathy and future directions for research and therapy. Diabetes Care. 8:290-299.

4. Greene, D. A. 1983. Metabolic abnormalities in diabetic peripheral nerve: relationship to impaired function. Metab. Clin. Exp. 32:118-123.

5. Winegrad, A. I., and D. A Simmons. 1983. Editorial. N. Engl. J. Med. 308:152-154.

6. Greene, D. A., S. A. Lattimer, and A. A. F. Sima. 1987. Sorbitol, phosphoinositides and the sodium-potassium ATPase in the pathogenesis of diabetic complications. N. Engl. J. Med. 316:599-606.

7. Chakrabarti, S., A. A. F. Sima, T. Nakajima, S. Yagihashi, and D. A. Greene. 1987. Aldose reductase in the BB-rat: isolation, immunological identification and localization in the retina and peripheral nerve. Diabetologia. 30:244-251.

8. Brismar, T., and A. A. F. Sima. 1981. Changes in nodal function in nerve fibers of the spontaneously diabetic BB-Wistar rat. Potential clamp analysis. Acta Physiol. Scand. 113:499-506.

9. Brismar, T., A A. F. Sima, and D. A. Greene. 1987. Reversible and irreversible nodal dysfunction in diabetic neuropathy. Ann. Neurol. 21:504-507.

10. Mackway, A. M., and D. A. Greene. 1986. Decreased myoinositol content and ( $\mathrm{Na}, \mathrm{K})$-ATPase activity in the superior cervical ganglion of the streptozotocin-diabetic rat, and its prevention by aldose reductase inhibition. Diabetes. 35:1106-1108.

11. Green, R. J., R. H. M. King, P. K. Thomas, and D. N. Baron. 1985. Sodium-potassium-ATPase activity in the dorsal root ganglia of rats with streptozotocin-induced diabetes. Diabetologia. 28:104-107.

12. Yorek, M. A., J. A. Dunlap, and B. H. Ginsberg. 1987. myoInositol metabolism in 41 A3 neuroblastoma cells: effects of high glucose and sorbitol levels. J. Neurochem. 48:53-61.

13. Morrison, A. D. 1984. Aortic smooth muscle metabolism: effect of polyol pathway inhibition. Clin. Res. 32:851A. (Abstr.)

14. Larson, R. E., and A. D. Morrison. 1986. Effect of insulin on endothelial cell myo-inositol metabolism. Diabetes. 35(Suppl. 1):12A. (Abstr.)

15. Pryce, T. D. 1983. On diabetic neuritis with a clinical and pathologic description of diabetic pseudo-tabes. Brain. 16:416-424.

16. Woltman, H. W., and R. M. Wilder. 1929. Diabetes mellitus: pathologic changes in the spinal cord and peripheral nerves. Arch. Intern. Med. 44:575-603.

17. Fagerberg, S. E. 1959. Diabetic neuropathy, a clinical and histological study on the significance of vascular affections. Acta Med. Scand. 164(Suppl. 345):1-97.

18. Olsson, Y., J. Säve-Söderbergh, P. Sourander, and L. Angervall. 1968. A pathoanatomical study of the central and peripheral nervous system in diabetes of early onset and long duration. Pathol. Eur. 3:62-79.

19. Budzilovich, G. N. 1970. Diabetic neuropathy complex. Virchows Arch. Abt. A Pathol. Anat. 350:105-122.

20. Dyck, P. J., J. L. Karnes, P. O'Brien, H. Okazaki, A. Lais, and J. Engelstad. 1986. The spatial distribution of fiber loss in diabetic polyneuropathy suggests ischemia. Ann. Neurol. 19:440-449.

21. Dyck, P. J., A. Lais, J. L. Karnes, P. O'Brien, R. Rizza. 1986. Fiber loss is primary and multifocal in sural nerves in diabetic polyneuropathy. Ann. Neurol. 19:425-439.

22. Vracko, R. 1982. A comparison of the microvascular lesions in diabetes mellitus with those of normal aging. J. Am. Geriatr. Soc. 30:201-205.

23. Williams, E., W. R. Timperley, J. D. Ward, and T. Duckworth. 1980. Electron microscopic studies of vessels in diabetic peripheral neuropathy. J. Clin. Pathol. 33:462-470.

24. Thomas, P. K., and R. G. Lascelles. 1965. Schwann-cell abnormalities in diabetic neuropathy. Lancet 1:1355-1357.

25. Thomas, P. K., and R. G. Lascelles. 1966. The pathology of diabetic neuropathy. $Q$. J. Med. 35:489-509.

26. Chopra, J. S., and T. Fannin. 1971. Pathology of diabetic neuropathy. J. Pathol. Bacteriol. 104:175-184.

27. Chopra, J. S., L. J. Hurwitz, and D. A. D. Montgomery. 1969. The pathogenesis of sural nerve changes in diabetes mellitus. Brain. 92:391-418.

28. Behse, F., F. Buchtal, and F. Carlsen. 1977. Nerve biopsy and conduction studies in diabetic neuropathy. J. Neurol. Neurosurg. Psychiatry. 40:1072-1082.

29. Bischoff, A. 1973. Ultrastructural pathology of peripheral nervous system in early diabetes. In Vascular and Neurologic Changes in Early Diabetes. R. A. Camerini-Davalos, and H. S. Cole, editors. Academic Press, Inc., New York. 441-449.

30. Dyck, P. J., W. R. Sherman, L. M. Hallcher, F. J. Service, P. C. O'Brien, L. A. Grina, P. J. Palumbo, and C. J. Swanson. 1980. Human diabetic endoneurial sorbitol, fructose and myo-inositol related to sural nerve morphometry. Ann. Neurol. 8:590-596.

31. Eng, G. D., N. Hung, and G. P. Agust. 1975. Nerve conduction velocity determination in juvenile diabetes. Mod. Probl. Paediatr. 12:213-219.

32. Kimura, J., T. Yamada, and N. P. Stevland. 1979. Distal slowing of motor nerve conduction velocity in diabetic polyneuropathy. $J$. Neurol. Sci. 42:291-302.

33. Hansen, S., and J. P. Ballantyne. 1977. Axonal dysfunction in the neuropathy of diabetes mellitus: a quantitative electrophysiological study. J. Neurol. Neurosurg. Psychiatry. 40:555-564.

34. Bischoff, A. 1968. Diabetische Neuropathie. Dtsch. Med. Wochensch. 93:237-241.

35. Bischoff, A. 1978. Report at the Aarhus University 50 year Jubilee Symposium: nervous system abnormalities and nervous disease in diabetes. Aarhus, Denmark.

36. Yasuda, H., and P. J. Dyck. 1987. Abnormalities of endoneurial microvessels and sural nerve pathology in diabetic neuropathy. Neurology. 37:20-28.

37. Yagihashi, S., and M. Matsumaja. 1979. Ultrastructural pathology of peripheral nerves in patients with diabetic neuropathy. Tohoku J. Exp. Med. 129:357-366.

38. Brismar, T. 1983. Diabetic neuropathy-functional abnormalities in the BB-rat. Metab. Clin. Exp. 32:112-117.

39. Sima, A. A. F., and T. Brismar. 1985. Reversible diabetic nerve dysfunction: structural correlates to electrophysiological abnormalities. Ann. Neurol. 18:21-29. 
40. Greene, D. A., S. Chakrabarti, S. A. Lattimer, and A. A. F. Sima. 1987. Role of sorbitol accumulation and myo-inositol depletion in paranodal swelling of large myelinated nerve fibers in the insulin deficient spontaneously diabetic Bio-breeding rat: reversal by insulin replacement, an aldose reductase inhibitor and myo-inositol. J. Clin. Invest. 79:1479-1485.

41. Sima, A. A. F., S. A. Lattimer, S. Yagihashi, and D. A. Greene. 1986. Axo-glial dysjunction. A novel structural lesion that accounts for poorly reversible slowing of nerve conduction in the spontaneously diabetic Bio-breeding rat. J. Clin. Invest. 77:474-484.

42. Greene, D. A., S. Yagihashi, S. A. Lattimer, and A. A. F. Sima. 1984. Nerve $\mathrm{Na}^{+}-\mathrm{K}^{+}$-ATPase, conduction and myo-inositol in the insulin deficient BB-rat. Am. J. Physiol. 247:E534-E539.

43. Rosenbluth, J. 1978. Glial membrane specializations in extraparanodal regions. J. Neurocytol. 7:709-719.

44. Sima, A. A. F., M. Bouchier, and H. Christensen. 1983. Axonal atrophy in sensory nerves of the diabetic BB-Wistar rat. A possible early correlate of human diabetic neuropathy. Ann. Neurol. 13:264272.

45. Pirart, J. 1978. Diabetes mellitus and its degenerative complications: a prospective study of 4,400 patients observed between 1947 and 1973. Diabetes Care. 1:168-188; 252-263.

46. National Diabetes Data Group. 1979. Classification and diagnosis of diabetes mellitus and other categories of glucose intolerance. Diabetes. 28:1039-1057.

47. Arezzo, J. C., and H. H. Schaumburg, and C. A. Peterson. 1983. Rapid screening for peripheral neuropathy: a field study with the Opticon. Neurology. 33:626-629.

48. Arezzo, J. C., H. H. Schaumburg, and C. Laudadio. 1986. Thermal sensitivity tester: device for quantitative assessment of thermal sense in diabetic neuropathy. Diabetes. 35:590-592.

49. Dyck, P. J., J. Karnes, A. Lais, E. P. Löfgren, and J. C. Stevens. 1984. Pathologic alterations of the peripheral nervous system of humans. In Peripheral Neuropathy. P. J. Dyck, P. K. Thomas, E. H. Lambert, and R. Bunge, editors. W. B. Saunders Co., Philadelphia. 760-870.

50. Dyck, P. J., J. Karnes, P. C. O’Brien, H. Nakuda, A. Lais, and P. Low. 1984. Spatial pattern of nerve fiber abnormalities indicative of pathologic mechanism. Am. J.Pathol. 117:225-238.

51. O'Neill, J. H., and R. W. Gilliatt. 1986. Myelin remodeling during axonal atrophy. Proceedings of the Tenth International Congress of Neuropathology, Stockholm, Sweden (Abstr.).

52. Dyck, P. J., J. Karnes, A. Lais, E. P. Lofgren, and J. C. Stevens. 1984. Pathologic alterations of the peripheral nervous system of humans. In Peripheral Neuropathy. P. J. Dyck, P. K. Thomas, E. H. Lambert, and R. Bunge, editors. W. B. Saunders Co., Philadelphia. 760-870.

53. Buchtal, F., A. Rosenfalck, and F. Behse. 1984. Sensory potential of normal and diseased nerves. In Peripheral Neuropathy. P. J. Dyck, P. K. Thomas, E. H. Lambert, and R. Bunge, editors. W. B. Saunders Co., Philadelphia. 981-1015.

54. Bril, V., A. A. F. Sima, M. R. Werb, and D. A. Greene. 1985. Motor electrophysiological studies in diabetic patients. Electroencephalogr. Clin. Neurophysiol. 61:S147.

55. Dyck, P. J., P. A. Low, M. F. Sparks, L. A. Herum, and J. L. Karnes. 1980. Effect of serum hyperosmolality on morphometry of healthy human sural nerves. J. Neuropathol. Exp. Neurol. 30:285-295.

56. Berthold, C.-H., I. Nilsson, and M. Rydmark. 1983. Axonal diameter and myelin sheath thickness in nerve fibers of the spinal root and the seventh lumbar nerve of the adult and developing cat. J. Anat. 136:483-493.

57. Vizoso, A D., and J. Z. Young. 1948. Internodal length and fiber diameter in developing and regenerating nerves. J. Anat. 82:110134.

58. Vizoso, A. D. 1950. The relationship between internodal length and growth in human nerves. J. Anat. 84:342-353.

59. Reske-Nielsen, E., and K. Lundbaeck. 1968. Pathological changes in the central and peripheral nervous system of young longterm diabetics. II. The spinal cord and peripheral nerves. Diabetologia. 4:34-43.

60. Said, G., G. Slama, and J. Selva. 1983. Progressive centripetal degeneration of axons in small fiber diabetic polyneuropathy. Brain. 106:791-807.

61. Sima, A. A. F. 1980. Peripheral neuropathy in the spontaneously diabetaic BB-Wistar rat. Acta Neuropathol. 51:223-232.

62. Sima, A. A. F., A. C. Larusso, and P. Thibert. 1982. Distal symmetric polyneuropathy in the spontaneously diabetic BB-Wistar rat. An ultrastructural and teased fiber study. Acta Neuropathol. 58:39-47.

63. Jakobsen, J. 1976. Axonal dwindling in early experimental diabetes. A study of cross sectioned nerves. Diabetologia. 12:539-546.

64. Greene, D. A., V. Bril, S. A. Lattimer, and A. A. F. Sima. 1987. Correction of myo-inositol depletion in diabetic human sural nerve by treatment with an aldose reductase inhibitor. Diabetes 36(Suppl. 1):86A. (Abstr.)

65. Mulvany, M. J. 1986. Vascular effects of altered sodium, potassium pump activity. Scand. J. Clin. Lab. Invest. 46:40-48. 